The final publication is available at Elsevier via https://doi.org/10.1016/j.jforecast.2018.07.005. (c) 2019. This manuscript version is made available under the CC-BY-NC-ND 4.0 license http://creativecommons.org/licenses/by-nc-nd/4.0/

\title{
Incentive compatibility in prediction markets: costly actions and external incentives
}

\author{
Chen $\mathrm{Di}^{\mathrm{a}}$, Stanko Dimitrov ${ }^{\mathrm{a}, *}$, Qi-Ming $\mathrm{He}^{\mathrm{a}}$ \\ ${ }^{a}$ Department of Management Sciences, \\ University of Waterloo, \\ Waterloo, ON N2L $3 G 1$.
}

\begin{abstract}
We consider a multi-round prediction market in which two agents, Alice and $B$ ob, are trading on an event which they may both take actions to influence its outcome. Existing literature assumes that when external incentives exist, there is no net difference between the cost of different actions agents may take outside of the prediction market. For example, it is the same cost for either Alice to work hard to complete the project, as it is for her to "loaf" and not work hard. In this work we consider a 2-round and later a 4-round setting in which agents' costs of external actions differ for each action. We show that when external action costs differ but are within a proper range, a prediction market is incentive compatible regardless of the initial market estimate, something that currently is not shown in the existing literature.
\end{abstract}

Keywords: prediction markets, external incentives, costly-actions, incentive compatibility, Decision making

\section{Background Introduction}

A prediction market is created to aggregate information from individuals about uncertain events of interest. For example, a prediction market like mechanism is known to better predict the financial value of soccer players in transfer markets Peeters (2018) than other standard forecasting methods. However, some decision makers are suspicious about using prediction markets in all settings, such as corporate settings. In corporate settings

\footnotetext{
*Corresponding author

Email addresses: cdi@uwaterloo.ca (Chen Di), sdimitro@uwaterloo.ca (Stanko Dimitrov), q7he@uwaterloo.ca (Qi-Ming He)
} 
managers are concerned that workers may be incentivized to take adverse actions within the workplace when a prediction market is present as opposed to when it is not present. One public event where external actions may influence a traded event is an election. Depending on the stakes within a prediction market, some voters may change their vote to maximize their total return both within and without the prediction market. The preceding argument, though theoretically valid, is yet to be shown to hold any merit in practice. In fact, prediction markets are used extensively to elicit and aggregate opinions on political events (Graefe et al., 2014; Hanea et al., 2017; Rothschild, 2015). It is generally assumed that agents who participate in a prediction market by trading may have superior information about the relevant event, but no direct control over the event outcome (e.g., one individual vote is likely to not sway an entire election). However, prediction markets are often used in situations where this assumption is violated (Chakraborty and Das, 2016) (e.g., student evaluations). In this study we consider the impact of external incentives on the efficacy of prediction markets, especially when acting on external incentives requires costly actions. Prediction markets that are deployed in corporate settings consist of a market maker, a center with whom all participants, agents, trade, that is present to facilitate trade and boost market liquidity. In our study, the market maker, is also the market participants' employer, does not want agents to take undesirable actions at work that may impact the outcome of the traded event.

There is substantial evidence that prediction markets can help produce forecasts on event outcomes with lower prediction errors than conventional forecast methods (Arrow et al., 2008). However, it is also possible that agents might bluff and deceive other players by not revealing their true beliefs, hoping to later correct the prediction probability and benefit later in the market (Chen et al., 2010; Dimitrov and Sami, 2008). In addition to bluffing to maximize prediction market payoff, an agent may also change her behavior outside of the prediction market to maximize her reward within the market as well as outside of the market. For example, employees might have incentives to "slack off" when working on a project just because their prediction market position is favorable to the project being delayed and would somehow benefit more in the prediction market by working less hard in the workplace. This may seem like a far-fetched idea, but not long ago the U.S. congress was worried that terrorists might have a higher incentive to actually conduct terrorist attacks if they trade in a relevant prediction market (Looney, 2004). Eventually these proposed "terrorist markets" were shut down by the U.S. congress due to these concerns. There are additional canonical real-world examples showing that having agents trade in a predic- 
tion market distorts the same agents' incentives within and outside of the prediction market (Chakraborty and Das, 2014), such as instructor prediction markets (Chakraborty et al., 2013). In fact, there is no ex post way to determine how the presence of a prediction market changes agents' probability estimates, without considering the equilibrium strategies of agents within prediction markets. In this study, we use equilibrium analysis to see

if prediction markets may indeed cause deviant agent behavior when agents' external actions to the market are costly.

In conducting equilibrium analysis with the presence of external incentives in prediction markets, we find that when external actions have asymmetric costs, one action costs more or less than another, then these asymmetries may actually lead agents to behave truthfully. Specifically, we determine the equilibrium strategies for two agents in a 2-round and later a 4round setting where the agents trade in a prediction market with a final value contingent on an event that the same two agents have a direct impact on the likelihood of the traded event. We prove that the final equilibrium strategy shows that participants will always take desirable actions/undesirable actions related to the project (work hard or "loaf") and be truthful when reporting in the prediction market, as they would have had the prediction market not existed. Our result is important in the face of concern decision makers have regarding a prediction market potentially inducing undesirable actions.

In the remainder of this document we introduce related work in Section 2 and define our general model in Section 3. We next show that when the cost of exerting high effort is positive, agents do not work hard, but are truthful in the prediction market in Section 4.2; similarly we show that when the cost of exerting high effort is negative, agents work hard and are sill truthful in the prediction market in Section 4.3. However, we show in Section 4.4 that when there is no cost for efforts, there is possibility that agents do not always work hard and they do not report truthfully in the prediction market. In Section 4.5 we extend our 2-round setting to 4 rounds and prove that our conclusion still holds. In Section 5 we conclude and discuss future research directions.

\section{Literature Review}

A large portion of research on prediction markets and scoring rules, a building block of the prediction markets we consider in our paper, does not consider outside incentives. Brier (1950) defines the quadratic scoring rule and implicitly assumes agents cannot influence the outcome of the predicted 
event (tomorrow's weather). Bickel (2007) compares three commonly used strictly proper scoring rules: quadratic, spherical and logarithmic scoring rules showing the logarithmic scoring rule is preferred when accounting for non-linear utilities and rank ordering. Merkle and Steyvers (2013) demonstrate that different strictly proper scoring rules yield considerably different rankings of forecasters based on their scoring rule scores. We, however, assume agents interact in a market scoring rule market proposed by Hanson (2002), derived from the difference of sequential scoring rules. Hanson's market scoring rule (MSR) incentivizes risk-neutral and myopic agents to truthfully reveal their probabilistic estimates by ensuring that truthful reporting maximizes an agent's expected payoffs, and is thereby said to be incentive compatible. Das (2008) implies that market making can serve as an effective trading strategy for individual agents who do not possess superior information but are willing to learn from prices. Unlike the work of Das (2008), we consider MSR prediction markets in which forward-looking agents may take costly external actions outside of the market to influence the likelihood of the traded event (for example, not work hard or vote for another candidate in an election). The agents in our setting are informed and do not simply learn from the traded prices.

It is generally assumed that the agents who participate in a prediction market by trading may have superior information about the traded event, but no direct control over the outcome as noted by Chakraborty and Das (2016). In their paper Chakraborty and Das (2016) show that if an agent does not participate in a prediction market, then they behave truthfully outside of the prediction market, but may change their outside actions as a function of their earlier prediction market report. The authors consider a voting game, in which the voting actions have equal costs and voting occurs after all agents concluded interacting in the prediction market. Unlike Chakraborty and Das (2016), we consider iterative interactions between agents, outside actions, and actions, reports, within the prediction market. In addition, and this is perhaps the most important difference we assume asymmetric cost of external actions, for example not voting is preferred to voting (there may be effort involved in voting that is saved when not voting in practice). Using the taxonomy of Chakraborty and Das (2016) one can say we consider both price manipulation (altering market price due to external rewards) and outcome manipulation (altering external actions based on market prices) in our study. As such we discuss both of these form of prediction market manipulation now. Similar to Chakraborty and Das (2016), other papers assume agents may influence the outcome of events traded in a prediction market or have some other incentive outside of the 
prediction market (Chakraborty and Das, 2014; Dimitrov and Sami, 2010; Huang and Shoham, 2014; Shi et al., 2009). Shi et al. (2009) indicate a potential downside of prediction markets is that they may incentivize agents to take undesirable actions, and prove that there exist principle-aligned prediction mechanisms that do not incentivize undesirable actions with an 'overpayment result.' In particular, unlike our work, Shi et al. (2009) do not use a market scoring rule mechanism, instead uses sequential scoring rules, and have linear subsidy (in the number of agents). Chakraborty and Das (2014) give a two-round example to understand when markets may be prone to manipulation due to different outside incentives and how much to trust the resulting prediction probabilities. However, Chakraborty and Das (2014), unlike our work, do not consider costly actions. Huang and Shoham (2014) assume profit-indifferent manipulators and propose a modification to market scoring rules in the form of trade limits, in order to reduce the extent of manipulation of prediction markets due to external incentives. However, the limitation of trade amounts may also interfere with eliciting agents' true beliefs (depending on a market's liquidity, bounded budgets may lead to agents not revealing all of their information in a market), a limitation we do not have in our work as we do not bound agents' budgets. Chen et al. (2014) employ a two-player market scoring rule setting where a manipulator with outside incentives trade first, followed by a truthful trader. We also have the two-player market setting but the two agents in our model are both strategic traders with outside incentives. Unlike the papers cited in this paragraph, we show that when non-myopic agents' expected payoffs not only consist of payoffs from the prediction market, but also include the costs of their related actions which decide the outcome of the market, then the quadratic scoring rule, used as the market reward mechanism, incentivizes agents to report truthfully in the prediction market and to take actions as if the prediction market was not present. We realize that the claims made regarding our contribution are very broad and as with any analytical result there are assumptions and inherent limitations regarding the generalizability of the result. We explicitly discuss the limitations and shortcoming of our result in Section 3, after presenting our model. We further discuss limitations of our result in Section 5. These discussions are presented not only for researchers to consider these limitations when deciding to apply our model and results, but also to identify future research directions. 


\section{Model Description}

In this section, we propose and describe our setting formally. In a company $C$, two employees are assigned to complete a time-limited project $E$ together, and we call them Alice and Bob. We consider each week as a round from the beginning of this project and the scheduled time for completing this project is $T$ weeks. As our model is in a multi-round setting, the number of rounds should be equal or larger than 2. In every round, Alice and Bob will separately decide whether to give high, later denoted as 1 , or low, later denoted as 0 , effort to project $E$ during each week. After $T$ rounds, Alice and Bob's total efforts will determine the likelihood of the project's success (e.g., meeting its scheduled delivery date). The project $E$ has binary outcome as $E$ occurs or $E$ does not occur. If the project succeeds by the end of $T$ rounds, we say $E$ occurs; if the project fails by the end of $T$ rounds, we say $E$ does not occur. At the end of every round, every high effort will bring some payoff scores (negative scores are equivalent to net costs and positive scores are equivalent to net rewards) to the player who exerted this effort; low efforts will not bring any payoff scores to the players. ${ }^{1}$ In our model effort is rewarded immediately after each round. This is a simplifying assumption, and need not necessarily hold in practice. In fact, in practice, a manager can tell if an employee is not working hard over time, and for ease of modeling, we assume this observation is immediate, but may not be so in practice. If reward is delayed, then this reward is appropriately discounted.

At the same time, in a prediction market, Alice and Bob also trade in security $F$ whose ultimate value is contingent on the outcome of $E$. We assume that the prediction market is a market scoring rule market, as we find is used in practice (PredictIt, 2014). If there is no related prediction market in $C$, then employees will be inspired to always devote high/low efforts to $E$ in order to gain maximal expected payoffs. However, when a prediction market contingent on $E$ exists, the employer may worry that employees will change their effort levels in order to benefit more through the rewards procured in the prediction market.

For every round $i(i=1, \ldots, T)$, Alice and Bob devote efforts $e_{A}^{(i)}$ and

\footnotetext{
${ }^{1}$ Payoff scores need not be linear in effort and the constant $\alpha$ is used to convert the functional form of effort to payoff scores, in order to be compared with scores earned in the prediction market. When payoff score is negative, high efforts bring net costs to agents; when payoff score is positive, high efforts bring net rewards to agents. The total scores earned from exerting efforts and reporting in the prediction market could be converted to some financial costs or rewards that will be given to the agents.
} 
$e_{B}^{(i)}$ to project $E$ and report prediction probabilities as $r_{A}^{(i)}$ and $r_{B}^{(i)}$ in the prediction market, respectively. When $E$ occurs, a report of $r_{A}^{(i)}$ earns Alice a net score $\rho_{s}^{(i)}\left(r_{A}^{(i)}\right)=s\left(E, r_{A}^{(i)}\right)-s\left(E, r_{B}^{(i-1)}\right)$, where $s$ is a proper scoring rule; when $E$ does not occur, the report earns Alice a net score $\rho_{f}^{(i)}\left(\bar{r}_{A}^{(i)}\right)=s\left(E, \bar{r}_{A}^{(i)}\right)-s\left(E, \bar{r}_{B}^{(i-1)}\right)$ instead $\left(\rho_{s}^{(i)}\left(r_{B}^{(i)}\right)\right.$ and $\rho_{f}^{(i)}\left(\bar{r}_{B}^{(i)}\right)$ are similarly defined). Note that $\bar{r}_{A}^{(i)}=1-r_{A}^{(i)}$. In the remainder of this document we will analogously define $\bar{w}=1-w$ for any variable $w$. In addition, $s(\cdot)$ is said to be a proper scoring rule if for a risk-neutral agent with belief $p$ and report $r$ on an event, then:

$$
\left.\frac{d}{d r}(p s(r)+\bar{p} s(\bar{r}))\right|_{r=p}=0,
$$

and

$$
\frac{d^{2}}{d r^{2}}(p s(r)+\bar{p} s(\bar{r})) \leq 0 .
$$

When an agent's score maximizing report is equal to her true belief, a proper scoring rule, and in turn a market scoring rule, is said to be incentive compatible. Here we assume that Alice (Bob) assumes the other player is myopic when she (he) sees his (her) previous prediction in the market. We also use $\nu_{A}\left(0<\nu_{A}<1\right)$ to describe Alice's impact on the likelihood of success of project $E$. We denote $h_{A}^{(i)}\left(h_{B}^{(i)}\right)$ as the accumulated number of high efforts exerted by Alice $(B \mathrm{ob})$ by the end of round i. $p_{A}^{(i)}\left(p_{B}^{(i)}\right)$ is the belief on the likelihood of $E$ occurring held by Alice $(B \circ b)$ in round $i$ before she (he) takes any actions in round $i . \pi_{A}^{(i)}\left(\pi_{B}^{(i)}\right)$ is the payoff earned from the current round, $i$, to the final round $T$ by Alice $(B \mathrm{ob})$ from exerting efforts towards $E$ and from making reports in the prediction market in all rounds $i$ through $T . I_{A}^{(i)}\left(I_{B}^{(i)}\right)$ is the system state that Alice (Bob) has formed in round $i$ after she (he) observes the most recent prediction probability and before she (he) takes any actions in round $i$. $\mathbb{E}\left[\pi_{A}^{(i)}\left(I_{A}^{(i)}, a_{A}^{(i)}\right)\right]\left(\mathbb{E}\left[\pi_{B}^{(i)}\left(I_{B}^{(i)}, a_{B}^{(i)}\right)\right]\right)$ is the expected payoff given the current system state $I_{A}^{(i)}\left(I_{B}^{(i)}\right)$ and the action set $a_{A}^{(i)}\left(a_{B}^{(i)}\right)$ that $A$ lice $(B o b)$ would take in round $i$; while $\mathbb{E}\left[\pi_{A}^{(i) *}\left(I_{A}^{(i)}\right)\right]\left(\mathbb{E}\left[\pi_{B}^{(i) *}\left(I_{B}^{(i)}\right)\right]\right)$ is the corresponding maximal expected payoff score for a given state $I_{A}^{(i)}\left(I_{B}^{(i)}\right)$ for Alice (Bob). $a_{A}^{(i) *}=\left(e_{A}^{(i) *}, r_{A}^{(i) *}\right)\left(a_{B}^{(i) *}=\left(e_{B}^{(i) *}, r_{B}^{(i) *}\right)\right)$ is the optimal action set $A$ lice $(B \mathrm{ob})$ takes in round $i$ in order to receive $\mathbb{E}\left[\pi_{A}^{(i) *}\left(I_{A}^{(i)}\right)\right]\left(\mathbb{E}\left[\pi_{B}^{(i) *}\left(I_{B}^{(i)}\right)\right]\right)$.

Figure 1 shows the dynamics of our model. We assume that in each round Alice first determines her effort level, $e_{A}^{(i)}$, then she makes a report 
in the prediction market, $r_{A}^{(i)}$; next, Bob determines the effort level he exerts in this round, $e_{B}^{(i)}$, and finally the round concludes by Bob reporting in the prediction market, $r_{B}^{(i)}$. However, during each round, each agent determines her (his) effort level and reported belief for this round simultaneously, though the actions exerted towards the project and belief reported in the prediction market are conducted sequentially. Alice's and Bob's reports in the prediction market are always common knowledge to both agents in all cases. Effort levels, however, are not common knowledge.

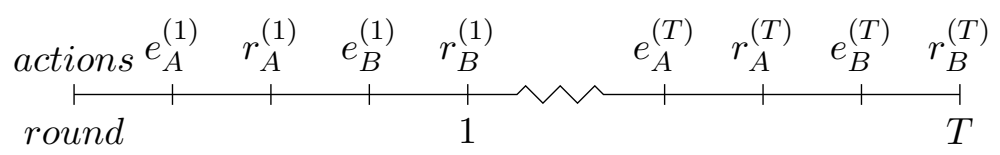

Figure 1: Model dynamics timeline

Given that in each round two risk-neutral forward-looking agents are maximizing their expected profits from the current round to the end of the project horizon, $T$, we can write down the Bellman equation to determine the payoff for each round, for each agent. Before we write down the equations for each round, we first define the payoffs each agent will receive in each round. In round $i$, Alice $(B \mathrm{ob})$ will receive $\rho_{e}^{(i)}\left(e_{A}^{(i)}\right)\left(\rho_{e}^{(i)}\left(e_{B}^{(i)}\right)\right)$ from devoting effort $e_{A}^{(i)}\left(e_{B}^{(i)}\right)$ to the project, and receive $p_{A}^{(i)} \cdot \rho_{s}^{(i)}\left(r_{A}^{(i)}\right)+\bar{p}_{A}^{(i)} \cdot \rho_{f}^{(i)}\left(\bar{r}_{A}^{(i)}\right)$ $\left(p_{A}^{(i)} \cdot \rho_{s}^{(i)}\left(r_{B}^{(i)}\right)+\bar{p}_{B}^{(i)} \cdot \rho_{f}^{(i)}\left(\bar{r}_{B}^{(i)}\right)\right)$ from reporting probability estimate $r_{A}^{(i)}\left(r_{B}^{(i)}\right)$ in the prediction market. As previously introduced, the value of the payoff function of exerted efforts, $\rho_{e}^{(i)}\left(e_{A}^{(i)}\right)\left(\rho_{e}^{(i)}\left(e_{B}^{(i)}\right)\right)$, is negative when high efforts bring net costs, and positive when high efforts bring net rewards. In our model we assume that the likelihood of $E$ occurring is determined by the total number of high efforts of all rounds, weighed by each of the agents' impact on determining the likelihood of $E$ :

$$
\frac{\nu_{A} \sum_{n=1}^{T} e_{A}^{(n)}+\left(1-\nu_{A}\right) \sum_{n=1}^{T} e_{B}^{(n)}}{T}
$$

For Alice in round $i$ before she takes any actions in this round, the number of accumulated high efforts exerted by her and by the end of round $i-1\left(h_{A}^{(i-1)}\right)$ is known to herself, but the number of accumulated high efforts exerted by $B$ ob and by the end of round $i-1\left(h_{B}^{(i-1)}\right)$ is not observed by her directly. However, $\sum_{n=i+1}^{T} \tilde{e}_{A}^{(n)}$ and $\sum_{n=i}^{T} \tilde{e}_{B}^{(n)}$ are all future efforts, and 
$e_{A}^{(i)}$ is the effort level decision she needs to make in the current round, $i$. For $B$ ob, before he takes any actions in round $i$, the situation is slightly different. Because Alice has already taken her actions in this round, the number of accumulated high efforts exerted by her is $h_{A}^{(i)}$ instead. To be more specific, we can write down the definitions of $p_{A}^{(i)}$ and $p_{B}^{(i)}$ as:

$$
p_{A}^{(i)}=\frac{\nu_{A}\left(h_{A}^{(i-1)}+e_{A}^{(i)}+\sum_{n=i+1}^{T} \tilde{e}_{A}^{(n)}\right)+\left(1-\nu_{A}\right)\left(h_{B}^{(i-1)}+\sum_{n=i}^{T} \tilde{e}_{B}^{(n)}\right)}{T},
$$

In equation (4a) we see the formal definition of $p_{A}^{(i)}$ as the total efforts of Alice, past and future, $h_{A}^{(i-1)}+\sum_{n=i}^{T} \tilde{e}_{A}^{(n)}$, plus the total efforts of $B$ ob, past and future, $h_{B}^{(i-1)}+\sum_{n=i}^{T} \tilde{e}_{B}^{(n)}$, all weighted by $\nu_{A}$. From Alice's perspective, she knows her past effort levels, thus $h_{A}^{(i-1)}$ is known and is some natural number between 0 and $i-1$. Similarly, as we will see in our analysis, Alice may infer Bob's effort levels from his reports in the prediction market, and again $h_{B}^{(i-1)}$ is known to $A$ lice. The $\tilde{e}_{A}^{(n)}$ and $\tilde{e}_{B}^{(n)}$ effort values, for $n \geq i+1$, are not necessarily binary, and are instead real numbers over $[0,1]$ to account for the fact that Alice's and Bob's equilibrium effort strategies are mixed. As effort levels are not common knowledge, we have to define how each agent interprets the reported probability of the other agent. One way to interpret reported probabilities is to use Bayesian updating, given current prior beliefs. However, as the probability of $E$ is dependent not only on market estimates but current and future effort levels, defining the Bayesian updating policy is quite convoluted. To simplify our analysis, we define $r_{B}(A)^{(i)}$ as Bob's estimate on the likelihood of $E$ up to and including round $i$ after observing Alice's last report. However, when $A$ lice makes the report in round $i, B$ ob has not yet taken actions in this round, the expectation of his effort value of round $i$ perceived by Alice is denoted as $\mathbb{E}_{A}\left[\tilde{e}_{B}^{(i)}\right]$. We similarly define $r_{A}(B)^{(i)}$ as Alice's estimate of the likelihood of $E$ 's occurring up to and including round $i$ after observing Bob's last report. We formally 
define the two notations below as:

$$
\begin{aligned}
& r_{B}(A)^{(i)}=\frac{\nu_{A} h_{A}^{(i)}+\left(1-\nu_{A}\right)\left(h_{B}^{(i-1)}+\mathbb{E}_{A}\left(\tilde{e}_{B}^{(i)}\right)\right)}{i}, \\
& r_{A}(B)^{(i)}=\frac{\nu_{A} h_{A}^{(i)}+\left(1-\nu_{A}\right) h_{B}^{(i)}}{i} .
\end{aligned}
$$

Here we assume that at the beginning of round $i$, Alice has no information she may use to predict $B$ ob's effort level of this round, which implies $\mathbb{E}_{A}\left(\tilde{e}_{B}^{(i)}\right)=0.5$ for any round $i$. Then the notations of $r_{B}(A)^{(i)}$ and $r_{A}(B)^{(i)}$ can be further defined as:

$$
\begin{aligned}
& r_{B}(A)^{(i)}=\frac{\nu_{A} h_{A}^{(i)}+\left(1-\nu_{A}\right)\left(h_{B}^{(i-1)}+0.5\right)}{i}, \\
& r_{A}(B)^{(i)}=\frac{\nu_{A} h_{A}^{(i)}+\left(1-\nu_{A}\right) h_{B}^{(i)}}{i} .
\end{aligned}
$$

Note that $r_{B}(A)^{(i)}=r_{A}^{(i)}$ and $r_{A}(B)^{(i)}=r_{B}^{(i-1)}$, as we are simply presenting how each of the agents interprets the observed prediction market probabilities of the other agent. In (4a) Alice's number of previous high efforts, $h_{A}^{(i-1)}$, is known to herself; and the number of Bob's previous high efforts, $h_{B}^{(i-1)}$, could not be observed directly but could be inferred using his last prediction probability $r_{B}^{(i-1)}$ as $h_{B}^{(i-1)}=\frac{(i-1) \cdot r_{B}^{(i-1)}-\nu_{A} h_{A}^{(i-1)}}{1-\nu_{A}}$. For (4b) the unobservable number of previous high efforts exerted by Alice could also be inferred using $r_{A}^{(i)}$ as $h_{A}^{(i)}=\frac{i r_{A}^{(i)}-\left(1-\nu_{A}\right)\left(h_{B}^{(i-1)}+0.5\right)}{\nu_{A}}$. By inserting $h_{B}^{(i-1)}=\frac{(i-1) r_{B}^{(i-1)}-\nu_{A} h_{A}^{(i-1)}}{1-\nu_{A}}$ and $h_{A}^{(i)}=\frac{i r_{A}^{(i)}-\left(1-\nu_{A}\right)\left(h_{B}^{(i-1)}+0.5\right)}{\nu_{A}}$ into (4a) and $(4 \mathrm{~b})$ separately, we can get a further expression of the agents' beliefs on the likelihood of $E$ 's occurrence during any round $i$ as:

$$
p_{A}^{(i)}=\frac{(i-1) r_{B}^{(i-1)}+\nu_{A}\left(e_{A}^{(i)}+\sum_{n=i+1}^{T} \tilde{e}_{A}^{(n)}\right)+\left(1-\nu_{A}\right) \sum_{n=i}^{T} \tilde{e}_{B}^{(n)}}{T},
$$

With the payoff scores collected in each round, each agent maximizes the current round's payoff scores plus the discounted future rounds' payoff 
scores.

$$
\begin{aligned}
\mathbb{E}\left[\pi_{A}^{(i) *}\left(I_{A}^{(i)}\right)\right]=\max _{\left(e_{A}^{(i)}, r_{A}^{(i)}\right)}\{\underbrace{}_{\text {discounted future payoff }} \underbrace{\delta\left[\pi_{A}^{(i+1) *}\right]}_{\text {effort payoff }}+\underbrace{\rho_{e}^{(i)}\left(e_{A}^{(i)}\right)}_{\text {prediction market payoff }} \\
+\underbrace{p_{A}^{(i)} \rho_{s}^{(i)}\left(r_{A}^{(i)}\right)+\bar{p}_{A}^{(i)} \rho_{f}^{(i)}\left(\bar{r}_{A}^{(i)}\right)}\}, \\
\mathbb{E}\left[\pi_{B}^{(i) *}\left(I_{B}^{(i)}\right)\right]=\max _{\left(e_{B}^{(i)}, r_{B}^{(i)}\right)}\left\{\delta \mathbb{E}\left[\pi_{B}^{(i+1) *}\right]+\rho_{e}^{(i)}\left(e_{B}^{(i)}\right)\right. \\
\left.+p_{B}^{(i)} \rho_{s}^{(i)}\left(r_{B}^{(i)}\right)+\bar{p}_{B}^{(i)} \rho_{f}^{(i)}\left(\bar{r}_{B}^{(i)}\right)\right\} .
\end{aligned}
$$

\section{Applicability of Developed Model}

Before moving to determining agents' equilibrium decisions in various settings, we will now discuss the applicability of the developed model. Recall that our paper is motivated by the fact prediction market agents participating in a market at work are paid for "doing their job," e.g., exerting high effort at work. Due to agents receiving compensation for exerting high effort, accounting for this compensation when determining an agent's equilibrium actions in a prediction market is necessary, and the purpose of this paper. However, as with most analytical work there are shortcomings not only with our model, which we will now discuss, but in turn with the conclusions which we discuss in Section 5.

One clear shortcoming with the developed model is that we are only considering two agents trading on a binary event ( $E$ either occurs or does not occur). As is noted in other research in prediction markets, results that hold for binary events do not always map to the $n$-nary outcome space (Karimi and Dimitrov, 2018). The reason binary events behave differently is change in one report is equivalent to a change in the opposite direction of the other report. With three or more outcomes, a change in one report, does not necessarily imply equal and opposite change in any other one report, instead change in aggregate multiple other reports.

Another clear shortcoming of the developed model is the agents. The agents are risk-neutral, something known not to be true in practice (Carlsson et al., 2005; Holt and Laury, 2002; Rosati and Hare, 2016). It is not clear how/if risk-attitudes impact agent's actions when exposed to external incentives. There is still scant research in risk-aversion in prediction markets without external incentives (Dimitrov et al., 2015; Karimi and Dimitrov, 2018; Ottaviani and Sørensen, 2007) let alone any research we are aware of 
on risk-aversion in the presence of external incentives. In addition to riskneutral agents, we also assume agents are homogeneous, in that they both either receive positive or negative cost for exerting high effort. In practice, the relative magnitude of agents' costs may impact their actions, especially when they have different signs.

The final significant shortcoming to our presented model is the stylized learning model for agents. The learning model allows us to have a tractable model, but results in agents not accounting for all of the other agent's future actions. This may not happen in practice, and may skew our results.

Even with all of the above shortcomings we think that the work presented in this paper is a necessary first step in further addressing external incentives in prediction markets. It is clear that agents participating in corporate prediction markets are paid for taking certain external actions while penalized for taking others. If this situation is not considered and modeled, then managers may still think that using prediction markets within a workplace is a bad idea. Granted we make multiple simplifying assumptions that lead to shortcomings in our model, but the results, as we will soon see, show that costly actions to act on external incentives may restore incentive compatibility in prediction markets. The results, are promising, and in addition to addressing the shortcoming outlined above as future work, our findings lend themselves to field or laboratory experiments to verify if costly actions do indeed restore incentive compatibility in prediction markets.

In the remainder of this document, we will determine the values of $\mathbb{E}\left[\pi_{A}^{(i) *}\left(I_{A}^{(i)}\right)\right]$ and $\mathbb{E}\left[\pi_{B}^{(i) *}\left(I_{B}^{(i)}\right)\right]$ and the equilibrium strategies of both agents.

\section{Result and Analysis}

This section first introduces one of the most commonly used scoring rules, the quadratic scoring rule, that will be used in all following cases. Then we give mathematical analysis of case 1, high efforts bring net cost to agents, case 2, high efforts bring net reward instead, and case 3, external incentives do not exist. We assume $T=2$ for the first three cases, however we discuss results for $T=4$ in case 4 .

In this work, we show that with external incentives (net payoff scores are given to exerted efforts), the quadratic scoring applied in the prediction market is incentive-compatible (agents will report truthfully in the prediction market). In fact, we prove that in a 2-round setting, agents will not take desirable actions (always exert high efforts) when high efforts bring net costs in case 1; and agents will take desirable actions (always exert high efforts) 
when high efforts bring net rewards in case 2. When external incentives exist, agents will behave as if the prediction market was not present, which indicates that the prediction market will not change agents' incentives outside of the market. However, when external incentives do not exist, we prove in case 3 that agents will have incentives to bluff (report untruthfully) in the prediction market when they are forward-looking. This result is aligned with the conclusion from previous work (Chen et al., 2010; Dimitrov and Sami, 2008). Finally we recreate case 2 in case 4 for the 4 -round setting.

\subsection{Application of the quadratic scoring rule}

In this section, we use a popular scoring rules, the quadratic scoring rule, as the reward mechanism in the prediction market. As we consider extreme reports in our results, agents report probability estimates of 0 or 1; we cannot use another, perhaps more popular, scoring rule, such as the logarithmic scoring rule. Then $s(E, r)$ introduced in Section 3 is defined as:

$$
\begin{aligned}
& s(E, r)=2 r-r^{2}-\bar{r}^{2}=-2 r^{2}+4 r-1 \\
& s(E, \bar{r})=2 \bar{r}-r^{2}-\bar{r}^{2}=-2 r^{2}+1
\end{aligned}
$$

$s(E, r)$ is a proper scoring rule as (1) and (2) are satisfied. We have already defined the scores earned by reporting in the prediction market in Section 3 as:

$$
\begin{aligned}
& \rho_{s}\left(r_{A}^{(i)}\right)=s\left(E, r_{A}^{(i)}\right)-s\left(E, r_{B}^{(i-1)}\right) \\
& \rho_{f}\left(\bar{r}_{A}^{(i)}\right)=s\left(E, \bar{r}_{A}^{(i)}\right)-s\left(E, \bar{r}_{B}^{(i-1)}\right) \\
& \rho_{s}\left(r_{B}^{(i)}\right)=s\left(E, r_{B}^{(i)}\right)-s\left(E, r_{A}^{(i)}\right) \\
& \rho_{f}\left(\bar{r}_{B}^{(i)}\right)=s\left(E, \bar{r}_{B}^{(i)}\right)-s\left(E, \bar{r}_{A}^{(i)}\right)
\end{aligned}
$$

Using $s(E, r)$ defined in (9), we can further write the scores as:

$$
\begin{aligned}
& \rho_{s}\left(r_{A}^{(i)}\right)=4 r_{A}^{(i)}-2\left(r_{A}^{(i)}\right)^{2}-4 r_{B}^{(i-1)}+2\left(r_{B}^{(i-1)}\right)^{2} \\
& \rho_{f}\left(\bar{r}_{A}^{(i)}\right)=-2\left(r_{A}^{(i)}\right)^{2}+2\left(r_{B}^{(i-1)}\right)^{2} \\
& \rho_{s}\left(r_{B}^{(i)}\right)=4 r_{B}^{(i)}-2\left(r_{B}^{(i)}\right)^{2}-4 r_{A}^{(i)}+2\left(r_{A}^{(i)}\right)^{2} \\
& \rho_{f}\left(\bar{r}_{B}^{(i)}\right)=-2\left(r_{B}^{(i)}\right)^{2}+2\left(r_{A}^{(i)}\right)^{2}
\end{aligned}
$$




\subsection{Case 1: High efforts bring net costs to agents}

In case 1 we assume that the two agents' efforts together decide the outcome of $E$ and the ultimate value of security $F$ but high efforts will bring net costs to the agents who exert them. In this setting, we assume the payoff function of exerted efforts to be $\rho_{e}(e)=-\alpha e^{2}(\alpha>0)$ :

$$
\begin{aligned}
& \rho_{e}\left(e_{A}^{(i)}\right)=-\alpha\left(e_{A}^{(i)}\right)^{2}, \\
& \rho_{e}\left(e_{B}^{(i)}\right)=-\alpha\left(e_{B}^{(i)}\right)^{2} .
\end{aligned}
$$

In this section we use the definition of Alice's (Bob's) belief on the likelihood $E$ 's final occurrence in each round when maximizing her (his) expected total payoff from Section 3, and apply the quadratic scoring rule introduced in section 4.1. From equations (12), (7) and (11), we know that $I_{A}^{(i)}=r_{B}^{(i-1)}$ and $I_{B}^{(i)}=r_{A}^{(i)}$ when deciding the values of $\mathbb{E}\left[\pi_{A}^{(i) *}\left(I_{A}^{(i)}\right)\right]$ and $\mathbb{E}\left[\pi_{B}^{(i) *}\left(I_{B}^{(i)}\right)\right]$, respectively. After inserting the payoff functions of exerted efforts (12), the belief on the likelihood of E's occurring (7), and the payoff functions in the prediction market (11) into the maximization equations (8) from the first round to the final round, we get the following maximization equations for Alice and Bob separately when $T=2$.

For agent Alice, based on equations (7), (8), and (11), we obtain

$$
\begin{aligned}
\mathbb{E}\left[\pi_{A}^{(1) *}\left(r_{B}^{(0)}\right)\right]= & \max _{\left(e_{A}^{(1)}, r_{A}^{(1)}\right)}\left\{\delta \mathbb{E}\left[\pi_{A}^{(2) *}\left(\tilde{r}_{B}^{(1)}\right)\right]-\alpha\left(e_{A}^{(1)}\right)^{2}\right. \\
& +2\left(\nu_{A}\left(e_{A}^{(1)}+\tilde{e}_{A}^{(2)}\right)+\left(1-\nu_{A}\right) \sum_{n=1}^{2} \tilde{e}_{B}^{(n)}\right)\left(r_{A}^{(1)}-r_{B}^{(0)}\right) \\
& \left.+2\left(\left(r_{B}^{(0)}\right)^{2}-\left(r_{A}^{(1)}\right)^{2}\right)\right\} \\
\mathbb{E}\left[\pi_{A}^{(2) *}\left(r_{B}^{(1)}\right)\right]= & \max _{\left(e_{A}^{(2)}, r_{A}^{(2)}\right)}\left\{-\alpha\left(e_{A}^{(2)}\right)^{2}\right. \\
& +2\left(r_{B}^{(1)}+\nu_{A} e_{A}^{(2)}+\left(1-\nu_{A}\right) \tilde{e}_{B}^{(2)}\right)\left(r_{A}^{(2)}-r_{B}^{(1)}\right) \\
& \left.+2\left(\left(r_{B}^{(1)}\right)^{2}-\left(r_{A}^{(2)}\right)^{2}\right)\right\} .
\end{aligned}
$$


For agent Bob, similarly, we obtain

$$
\begin{aligned}
& \mathbb{E}\left[\pi_{B}^{(1) *}\left(r_{A}^{(1)}\right)\right]=\max _{\left(e_{B}^{(1)}, r_{B}^{(1)}\right)}\left\{\delta \mathbb{E}\left[\pi_{B}^{(2) *}\left(\tilde{r}_{A}^{(2)}\right)\right]-\alpha\left(e_{B}^{(1)}\right)^{2}\right. \\
& +2\left(r_{A}^{(1)}+\nu_{A} \tilde{e}_{A}^{(2)}+\left(1-\nu_{A}\right)\left(e_{B}^{(1)}+\tilde{e}_{B}^{(2)}-0.5\right)\right) \\
& \left.\cdot\left(r_{B}^{(1)}-r_{A}^{(1)}\right)+2\left(\left(r_{A}^{(1)}\right)^{2}-\left(r_{B}^{(1)}\right)^{2}\right)\right\} \text {, } \\
& \mathbb{E}\left[\pi_{B}^{(2) *}\left(r_{A}^{(2)}\right)\right]=\max _{\left(e_{B}^{(2)}, r_{B}^{(2)}\right)}\left\{-\alpha\left(e_{B}^{(2)}\right)^{2}\right. \\
& +2\left(2 r_{A}^{(2)}+\left(1-\nu_{A}\right)\left(e_{B}^{(2)}-0.5\right)\right)\left(r_{B}^{(2)}-r_{A}^{(2)}\right) \\
& \left.+2\left(\left(r_{A}^{(2)}\right)^{2}-\left(r_{B}^{(2)}\right)^{2}\right)\right\} \text {. }
\end{aligned}
$$

From the time-line we know that Alice is the first agent to take actions in round 1. Although for Alice in round 1, $\tilde{r}_{B}^{(1)}, \tilde{e}_{A}^{(2)}, \tilde{e}_{B}^{(1)}$ and $\tilde{e}_{B}^{(2)}$ are values for future actions, which are not known to Alice now, but could be inferred by her, for agents are assumed to be rational, forward-looking and strategic in our model. To be more specific, Alice can play the whole game in her mind knowing that both agents want to maximize the expected scores earned from the current round to the final round in any round, and infer future optimal actions after exerting effort $e_{A}^{(1)}$ and making report $r_{A}^{(1)}$ in round 1 . Following this logic, we can use backwards induction to solve this problem, i.e., determine the values of (13) and (14).

Define $f_{B}^{(i)}\left(f_{A}^{(i)}\right)$ as the function of Bob's (Alice's) expected payoff scores earned from the current round $i$ and $f_{B}^{(i) *}\left(f_{A}^{(i) *}\right)$ is the corresponding optimal function value. First, we consider agent $B$ ob in round 2. For given $r_{A}^{(2)}$ by Alice in the second round (which is known to Bob), if Bob's action is $a_{B}^{(2)}=\left(e_{B}^{(2)}, r_{B}^{(2)}\right)$, then we have $f_{B}^{(2)}=\mathbb{E}\left[\pi_{B}^{(2)}\left(r_{A}^{(2)}, a_{B}^{(2)}\right)\right]$ as:

$$
\begin{aligned}
f_{B}^{(2)}= & -\alpha\left(e_{B}^{(2)}\right)^{2}-2\left(r_{B}^{(2)}\right)^{2}+2\left(r_{A}^{(2)}\right)^{2} \\
& +2\left(\left(1-\nu_{A}\right)\left(e_{B}^{(2)}-0.5\right)+2 r_{A}^{(2)}\right)\left(r_{B}^{(2)}-r_{A}^{(2)}\right) .
\end{aligned}
$$

To find Bob's optimal action $a_{B}^{(2) *}=\left(e_{B}^{(2) *}, r_{B}^{(2) *}\right)$, which maximizes $f_{B}^{(2)}$, in the feasible region $0 \leq e_{B}^{(2)}, r_{B}^{(2)} \leq 1$, we first find all the feasible KarushKuhn-Tucker (KKT) points of $f_{B}^{(2)}$ and then find the optimal solution. 
Lemma 1. Assume that $\alpha>\frac{\left(1-\nu_{A}\right)^{2}}{2}$. Then $f_{B}^{(2)}$ has one feasible $K K T$ point:

$$
\left(e_{B}^{(2)}, r_{B}^{(2)}\right)= \begin{cases}(0,0) & \text { if } r_{A}^{(2)} \leq\left(1-\nu_{A}\right) / 4 \\ \left(0, r_{A}^{(2)}-\left(1-\nu_{A}\right) / 4\right) & \text { if } r_{A}^{(2)}>\left(1-\nu_{A}\right) / 4\end{cases}
$$

equivalently we write: $\left(e_{B}^{(2)}, r_{B}^{(2)}\right)=\left(0, \max \left\{0, r_{A}^{(2)}-\frac{1}{4}\left(1-\nu_{A}\right)\right\}\right)$. 


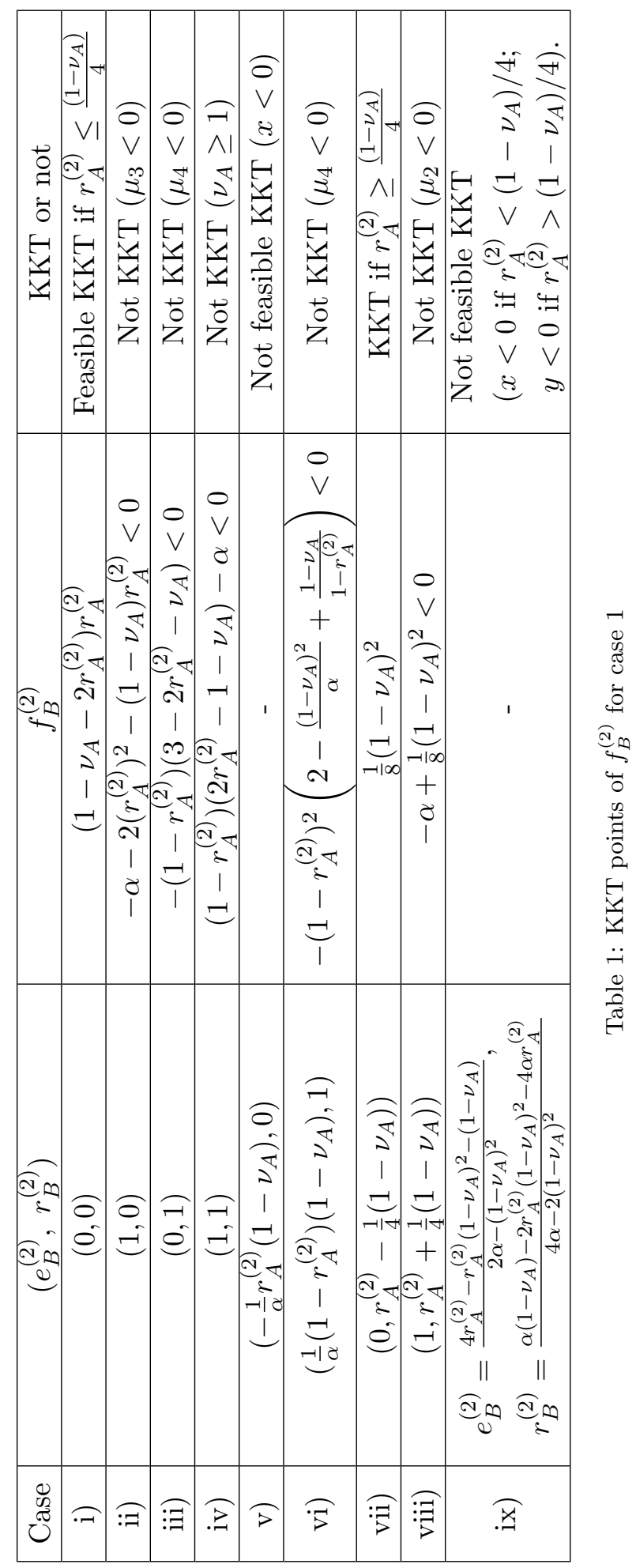


Proof. For notational convenience, in this proof, we let $x=e_{B}^{(2)}$ and $y=r_{B}^{(2)}$. The equations for the KKT points can be derived based on the function $f_{B}^{(2)}$ and the constraints $0 \leq x, y \leq 1$, which can be written explicitly as four inequality: $-x \leq 0, x-1 \leq 0,-y \leq 0$, and $y-1 \leq 0$. Correspondingly, four Lagrangian multipliers $\mu_{1}, \mu_{2}, \mu_{3}$, and $\mu_{4}$ are defined. By equation (4a) and routine calculations, the equations for KKT points are:

$$
\begin{aligned}
& -2 \alpha x+2 y\left(1-\nu_{A}\right)-2 r_{A}^{(2)}\left(1-\nu_{A}\right)+\mu_{1}-\mu_{2}=0 \\
& -4 y+2 x\left(1-\nu_{A}\right)+2\left(2 r_{A}^{(2)}-0.5\left(1-\nu_{A}\right)\right)+\mu_{3}-\mu_{4}=0 \\
& x \mu_{1}=(x-1) \mu_{2}=y \mu_{3}=(y-1) \mu_{4}=0 \\
& 0 \leq x \leq 1,0 \leq y \leq 1 ; \mu_{1} \geq 0, \mu_{2} \geq 0, \mu_{3} \geq 0, \mu_{4} \geq 0
\end{aligned}
$$

To find all the KKT points, we have and only have to consider the following nine cases: i) $x=y=0$; ii) $x=1$ and $y=0$; iii) $x=0$ and $y=1$; iv) $x=y=1$; v) $0<x<1$ and $y=0$; vi) $0<x<1$ and $y=1$; vii) $x=0$ and $0<y<1$; viii) $x=1$ and $0<y<1$; and ix) $0<x, y<1$. For each case, we find $\left\{x, y, \mu_{1}, \mu_{2}, \mu_{3}, \mu_{4}\right\}$ satisfying the above equations. In the end, only cases i) and vii) give feasible KKT points. Results are presented in Table 1. Reasons for why some cases do not have a KKT point are presented in the last column in Table 1. We also note that for non-feasible KKT points, the function $f_{B}^{(2)}$ is not given in Table 1 , since it is not used. Details are omitted.

We note that it is reasonable to assume $\alpha>\frac{\left(1-\nu_{A}\right)^{2}}{2}$. As $\nu_{A}$ will likely be some value around $\frac{1}{2}$ assuming $A$ lice and $B$ ob have new equal attribution to the project then the amount paid for the effort is greater than $\frac{1}{8}$ per round.

For the case with $r_{A}^{(2)} \geqslant \frac{1}{4}\left(1-\nu_{A}\right)$, we have the following results.

Lemma 2. If $\alpha>\frac{\left(1-\nu_{A}\right)^{2}}{2}$ and $r_{A}^{(2)} \geqslant \frac{1}{4}\left(1-\nu_{A}\right)$, then $\left(e_{B}^{(2) *}, r_{B}^{(2) *}\right)=\left(0, r_{A}^{(2)}-\right.$ $\left.\frac{1}{4}\left(1-\nu_{A}\right)\right)$ and $\mathbb{E}\left[\pi_{B}^{(2) *}\left(r_{A}^{(2)}\right)\right]=\frac{1}{8}\left(1-\nu_{A}\right)^{2}$.

Proof. The proof is similar to that of Lemma 1 . If $\frac{1}{4}\left(1-\nu_{A}\right) \leqslant r_{A}^{(2)}$, by comparing the values of $f_{B}^{(2)}$ of feasible KKT points in Table 1 , we find that $f_{B}^{(2) *}$ is attained as $f_{B}^{(2) *}=\frac{1}{8}\left(1-\nu_{A}\right)^{2}$ when $\left(e_{B}^{(2) *}, r_{B}^{(2) *}\right)=\left(0, r_{A}^{(2)}-\frac{1}{4}\left(1-\nu_{A}\right)\right)$. So Lemma 2 is true.

Based on Lemma 1 and Lemma 2, we find the optimal action for Bob in the second round. 
Theorem 1. If high efforts bring net costs and $\alpha>\frac{\left(1-\nu_{A}\right)^{2}}{2}$, then

$$
\begin{gathered}
\left(e_{B}^{(2) *}, r_{B}^{(2) *}\right)= \begin{cases}(0,0), & \text { if } r_{A}^{(2)} \leqslant \frac{1}{4}\left(1-\nu_{A}\right) ; \\
\left(0, r_{A}^{(2)}-\frac{1}{4}\left(1-\nu_{A}\right)\right), & \text { if } r_{A}^{(2)} \geqslant \frac{1}{4}\left(1-\nu_{A}\right),\end{cases} \\
f_{B}^{(2) *}= \begin{cases}-2\left(r_{A}^{(2)}\right)^{2}+\left(1-\nu_{A}\right) r_{A}^{(2)}, & \text { if } 0 \leqslant r_{A}^{(2)} \leqslant \frac{1}{4}\left(1-\nu_{A}\right) ; \\
\frac{1}{8}\left(1-\nu_{A}\right)^{2}, & \text { if } \frac{1}{4}\left(1-\nu_{A}\right) \leqslant r_{A}^{(2)} \leqslant 1 .\end{cases}
\end{gathered}
$$

Proof. First, we show that the function $f_{B}^{(2)}$ is concave in $\left(e_{B}^{(2)}, r_{B}^{(2)}\right)$. By routine calculations, we obtain the Hessian matrix of $f_{B}^{(2)}$ as:

$$
\nabla^{2} f_{B}^{(2)}\left(e_{B}^{(2)}, r_{B}^{(2)}\right)=\left[\begin{array}{ll}
\frac{\partial^{2} f_{B}^{(2)}}{\partial\left(e_{B}^{(2)}\right)^{2}} & \frac{\partial^{2} f_{B}^{(2)}}{\partial e_{B}^{(2)} \partial r_{B}^{(2)}} \\
\frac{\partial^{2} f_{B}^{(2)}}{\partial r_{B}^{(2)} \partial e_{B}^{(2)}} & \frac{\partial^{2} f_{B}^{(2)}}{\partial\left(r_{B}^{(2)}\right)^{2}}
\end{array}\right]=\left[\begin{array}{lr}
-2 \alpha & 2\left(1-\nu_{A}\right) \\
2\left(1-\nu_{A}\right) & -4
\end{array}\right]
$$

Since the sum of the diagonal elements of the Hessian matrix is negative (i.e., $-2 \alpha-4<0$ ) and the determinant of the Hessian matrix is positive (i.e., $\left.8 \alpha-4\left(1-\nu_{A}\right)^{2}>0\right)$, the two eigenvalues of the Hessian matrix must have the same sign, which has to be negative. Thus, the Hessian matrix is strictly negative definite. Therefore, function $f_{B}^{(2)}$ is concave. Then the optimal solution has to be the KKT point that maximizes $f_{B}^{(2)}$ or a boundary point of the feasible region. To find the optimal solution, we consider two cases, each has a unique KKT point.

If $r_{A}^{(2)} \leq\left(1-\nu_{A}\right) / 4$, there is only one feasible KKT point: $(0,0)$. Although there is no other feasible KKT point to be considered for optimality, we have to compare $f_{B}^{(2)}(0,0)$ with that of the all other feasible boundary solutions listed in Table 1. By routine calculations, it can be verified that other solutions are either infeasible or their $f_{B}^{(2)}$ is smaller than that of $(0,0)$. Therefore, $(0,0)$ is the optimal solution for this case.

If $r_{A}^{(2)}>\left(1-\nu_{A}\right) / 4$, there is only one KKT point: $\left(0, r_{A}^{(2)}-\left(1-\nu_{A}\right) / 4\right)$. Similar to the above case, it can be shown that $\left(0, r_{A}^{(2)}-\left(1-\nu_{A}\right) / 4\right)$ is the optimal solution for this case.

The corresponding $f_{B}^{(2) *}$ can be calculated accordingly and is given in Table 1.

Now, we consider Alice's expected payoff score in round $i=2$. We know from Theorem 1 that $e_{B}^{(2) *}=0$ no matter what value $r_{A}^{(2)}$ is. After 


\begin{tabular}{|c|c|c|c|}
\hline Cases & $\left(e_{A}^{(2)}, r_{A}^{(2)}\right)$ & $f_{A}^{(2)}$ & KKT or not \\
\hline i) & $(0,0)$ & 0 & Not KKT $\left(\mu_{3}<0\right)$ \\
\hline ii $)$ & $(1,0)$ & $-2 \nu_{A} r_{B}^{(1)}-\alpha<0$ & Not KKT $\left(\mu_{2}<0\right)$ \\
\hline iii $)$ & $(0,1)$ & $-\left(1-r_{B}^{(1)}\right)^{2}<0$ & Not KKT $\left(\mu_{1}<0\right)$ \\
\hline iv $)$ & $(1,1)$ & $2\left(1-\nu_{A}\right)\left(r_{B}^{(1)}-1\right)-\alpha<0$ & Not KKT $\left(\mu_{4}<0\right)$ \\
\hline v) & $\left(-\frac{\nu_{A} r_{B}^{(1)}}{\alpha}, 0\right)$ & - & Not feasible KKT $\left(e_{A}^{(2)}<0\right)$ \\
\hline vi $)$ & $\left(\frac{\nu_{A}\left(1-r_{B}^{(1)}\right)}{\alpha}, 1\right)$ & $2\left(r_{B}^{(1)}-1\right)+\frac{\nu_{A}^{2}\left(r_{B}^{(1)}-1\right)^{2}}{\alpha}<0$ & Not KKT $\left(\mu_{4}<0\right)$ \\
\hline vii $)$ & $\left(0, \frac{1}{2} r_{B}^{(1)}\right)$ & $\frac{1}{2}\left(r_{B}^{(1)}\right)^{2}$ & Feasible KKT \\
\hline viii) & $\left(1, \frac{1}{2}\left(r_{B}^{(1)}+\nu_{A}\right)\right)$ & $-\alpha+\frac{1}{2} \nu_{A}^{2}-r_{B}^{(1)} \nu_{A}+\frac{1}{2}\left(r_{B}^{(1)}\right)^{2}$ & Not KKT $\left(\mu_{2}<0\right)$ \\
\hline ix $)$ & $e_{A}^{(2)}=\frac{r_{B}^{(1)}\left(\nu_{A}-2\right)}{2 \alpha-\nu_{A}^{2}}<0$ & - & Not Feasible KKT $\left(e_{A}^{(2)}<0\right)$ \\
& $r_{A}^{(2)}=\frac{r_{B}^{(1)}\left(\alpha-\nu_{A}^{2}\right)}{2 \alpha-\nu_{A}^{2}}$ & & \\
\hline
\end{tabular}

Table 2: KKT points of $f_{A}^{(2)}$ in case 1

inserting $\tilde{e}_{B}^{(2)}=e_{B}^{(2) *}=0$ into the expression of $f_{A}^{(2)}=\mathbb{E}\left[\pi_{A}^{(2)}, r_{B}^{(1)}, a_{A}^{(2)}\right]$, where $a_{A}^{(2)}=\left(e_{A}^{(2)}, r_{A}^{(2)}\right)$, we obtain:

$$
\begin{aligned}
f_{A}^{(2)}=-\alpha\left(e_{A}^{(2)}\right)^{2} & +2\left(r_{B}^{(1)}+\nu_{A} e_{A}^{(2)}\right)\left(r_{A}^{(2)}-r_{B}^{(1)}\right) \\
& +2\left(\left(r_{B}^{(1)}\right)^{2}-\left(r_{A}^{(2)}\right)^{2}\right) .
\end{aligned}
$$

Lemma 3. Assume that $\alpha>\frac{\nu_{A}^{2}}{2}$. Then $f_{A}^{(2)}$ has a unique feasible $K K T$ point $\left(0, \frac{1}{2} r_{B}^{(1)}\right)$.

Proof. The proof is similar to that of Lemma 1. The system of equations used in the proof is given as follows (note: $x=e_{A}^{(2)}$ and $y=r_{A}^{(2)}$ ):

$$
\begin{aligned}
& -2 \alpha x+2 \nu_{A}\left(y-r_{B}^{(1)}\right)+\mu_{1}-\mu_{2}=0 \\
& -4 y+2\left(r_{B}^{(1)}+\nu_{A} x\right)+\mu_{3}-\mu_{4}=0 ; \\
& x \mu_{1}=(x-1) \mu_{2}=y \mu_{3}=(y-1) \mu_{4}=0 \\
& 0 \leq x \leq 1,0 \leq y \leq 1 ; \mu_{1} \geq 0, \mu_{2} \geq 0, \mu_{3} \geq 0, \mu_{4} \geq 0 .
\end{aligned}
$$

We find all the feasible KKT points from all the potential KKT points, which results in only one feasible KKT point. The results are shown in Table 2. Details are omitted. 
With Lemma 3 we introduce an additional bound with $\alpha>\frac{\nu_{A}^{2}}{2}$. As $\nu_{A} \in(0,1)$ we know that the lower bound on $\alpha$ is at most $\frac{1}{2}$, however Bob might not be involved in the project with $\nu_{A}$ so high. As such, if we consider more realistic values of $\nu_{A} \in[0.25,0.75]$, then the lower bound drops at most 0.28125 or lower. This lower bound on $\alpha$ is not very restrictive as $\alpha \in(0,2]$.

Theorem 2. If high efforts bring net costs and $\alpha>\frac{\nu_{A}^{2}}{2}$, then Alice's optimal action set in round 2 is $\left(e_{A}^{(2) *}, r_{A}^{(2) *}\right)=\left(0, \frac{1}{2} r_{B}^{(1)}\right)$, and her optimal expected payoff score of round 2 is $\mathbb{E}\left[\pi_{A}^{(2) *}\left(r_{B}^{(1)}\right)\right]=\frac{1}{2}\left(r_{B}^{(1)}\right)^{2}$.

Proof. To find the optimal function value $f_{A}^{(2) *}$, similar to Theorem 1, we need to check the Hessian of function $f_{A}^{(2)}$ :

$$
\nabla^{2} f_{A}^{(2)}\left(e_{A}^{(2)}, r_{A}^{(2)}\right)=\left[\begin{array}{ll}
\frac{\partial^{2} f_{A}^{(2)}}{\partial\left(e_{A}^{(2)}\right)^{2}} & \frac{\partial^{2} f_{A}^{(2)}}{\partial e_{A}^{(2)} \partial r_{A}^{(2)}} \\
\frac{\partial^{2} f_{A}^{(2)}}{\partial r_{A}^{(2)} \partial e_{A}^{(2)}} & \frac{\partial^{2} f_{A}^{(2)}}{\partial\left(r_{A}^{(2)}\right)^{2}}
\end{array}\right]=\left[\begin{array}{cc}
-2 \alpha & 2 \nu_{A} \\
2 \nu_{A} & -4
\end{array}\right] .
$$

We find that $f_{A}^{(2)}$ is a concave function. By Lemma 3 , there is only one candidate for our maximization, which is $\left(0, r_{B}^{(1)} / 2\right)$. Therefore, $\left(0, r_{B}^{(1)}\right) / 2$ is the optimal solution.

Next, we consider Bob in round 1. From Theorem 1 and Theorem 2 we have $e_{A}^{(2) *}=e_{B}^{(2) *}=0$. Knowing $\tilde{e}_{A}^{(2)}=\tilde{e}_{B}^{(2)}=0$, we get the expression of $f_{B}^{(1)}, B$ ob's expected cost in round 1 for action $a_{B}^{(1)}=\left(e_{B}^{(1)}, r_{B}^{(1)}\right)$, as:

$$
\begin{aligned}
f_{B}^{(1)}=-\alpha & \cdot\left(e_{B}^{(1)}\right)^{2}+2\left(r_{A}^{(1)}+\left(e_{B}^{(1)}-0.5\right)\left(1-\nu_{A}\right)\right)\left(r_{B}^{(1)}-r_{A}^{(1)}\right) \\
+ & 2\left(\left(r_{A}^{(1)}\right)^{2}-\left(r_{B}^{(1)}\right)^{2}\right) .
\end{aligned}
$$

Lemma 4. If $\alpha>\max \left\{\frac{\left(1-\nu_{A}\right)^{2}}{2}, \frac{\nu_{A}^{2}}{2}\right\}$, then

$$
\begin{gathered}
\left(e_{B}^{(2) *}, r_{B}^{(2) *}\right)= \begin{cases}(0,0), & \text { if } 0 \leqslant r_{B}^{(1)} \leqslant \frac{1}{2}\left(1-\nu_{A}\right) ; \\
\left(0, r_{A}^{(2)}-\frac{1}{4}\left(1-\nu_{A}\right)\right), & \text { if } \frac{1}{2}\left(1-\nu_{A}\right) \leqslant r_{B}^{(1)} \leqslant 1,\end{cases} \\
f_{B}^{(2) *}= \begin{cases}-2\left(r_{A}^{(2)}\right)^{2}+\left(1-\nu_{A}\right) r_{A}^{(2)}, & \text { if } 0 \leqslant r_{B}^{(1)} \leqslant \frac{1}{2}\left(1-\nu_{A}\right) ; \\
\frac{1}{8}\left(\nu_{A}-1\right)^{2}, & \text { if } \frac{1}{2}\left(1-\nu_{A}\right) \leqslant r_{B}^{(1)} \leqslant 1 .\end{cases}
\end{gathered}
$$


Proof. According to Theorem 2, we know that $r_{A}^{(2) *}=\frac{1}{2} r_{B}^{(1)}$. Together with the statement of Theorem 1, we infer Lemma 4.

We now move to Bob's optimal actions in the first round. We find that $B$ ob will exert low effort and report as 0 in round 1 no matter what report Alice makes in round 1.

Theorem 3. If high efforts bring net costs, $\alpha>\max \left\{\frac{\left(1-\nu_{A}\right)^{2}}{2}, \frac{\nu_{A}^{2}}{2}\right\}$, and $0<\nu_{A}<\frac{1}{3}$, then Bob's optimal action set in round 1 is $\left(e_{B}^{(1) *}, r_{B}^{(1) *}\right)=(0,0)$, and his expected total payoff score from this round to the final round is $\mathbb{E}\left[\pi_{B}^{(1) *}\left(r_{A}^{(1)}\right)\right]=r_{A}^{(1)}\left(1-\nu_{A}\right)$.

Proof. According to the definitions of $\mathbb{E}\left[\pi_{B}^{(1)}\left(r_{A}^{(1)}, a_{B}^{(1)}\right)\right], f_{B}^{(1)}$ and $f_{B}^{(2) *}$, we know that

$$
\mathbb{E}\left[\pi_{B}^{(1)}\left(r_{A}^{(1)}, a_{B}^{(1)}\right)\right]=f_{B}^{(1)}+\delta f_{B}^{(2) *}
$$

and $f_{B}^{(2) *}$ is a fixed value for a given $r_{A}^{(2) *}$. From Lemma 4 we know that if $r_{B}^{(1)} \leqslant \frac{1}{2}\left(1-\nu_{A}\right)$, then $\left(e_{B}^{(2) *}, r_{B}^{(2) *}\right)=(0,0)$ and $f_{B}^{(2) *}=-\frac{1}{2}\left(r_{B}^{(1)}\right)^{2}+\frac{1}{2}(1-$ $\left.\nu_{A}\right) r_{B}^{(1)}$; if $r_{B}^{(1)} \geqslant \frac{1}{2}\left(1-\nu_{A}\right)$, then $\left(e_{B}^{(2) *}, r_{B}^{(2) *}\right)=\left(0, r_{A}^{(2)}-\frac{1}{4}\left(1-\nu_{A}\right)\right)$ and $f_{B}^{(2) *}=\frac{1}{8}\left(1-\nu_{A}\right)^{2}$.

We first consider the case in which $f_{B}^{(2) *}$ is a function of $r_{B}^{(1)}$ as $f_{B}^{(2) *}=$ $-\frac{1}{2}\left(r_{B}^{(1)}\right)^{2}+\frac{1}{2}\left(1-\nu_{A}\right) r_{B}^{(1)}$, if $r_{B}^{(1)} \leqslant \frac{1}{2}\left(1-\nu_{A}\right)$. Under this condition, $f_{B}^{(1)}+$ $\delta f_{B}^{(2) *}$ is a concave function if $\alpha>\max \left\{\frac{\left(1-\nu_{A}\right)^{2}}{2}, \frac{\nu_{A}^{2}}{2}\right\}$. Similar to Theorems 1 and 2 , we find and compare the KKT points of $f_{B}^{(1)}+\delta \cdot f_{B}^{(2) *}$. It turns out that $f_{B}^{(1)}+\delta f_{B}^{(2) *}$ is maximized at $\left(e_{B}^{(1)}, r_{B}^{(1)}\right)=(0,0)$ with value $r_{A}^{(1)}\left(1-\nu_{A}\right)$. Details are omitted. (Note: In the rest of the paper, we will not give all the details about KKT points in our proofs.)

Second, we discuss the case in which $f_{B}^{(2) *}$ is a constant as $f_{B}^{(2) *}=\frac{1}{8}\left(\nu_{A}-\right.$ $1)^{2}$ if $\frac{1}{2}\left(1-\nu_{A}\right) \leqslant r_{B}^{(1)} \leqslant 1$. Under this condition, $f_{B}^{(1)}+\delta f_{B}^{(2) *}$ is also a concave function if $\alpha>\max \left\{\frac{\left(1-\nu_{A}\right)^{2}}{2}, \frac{\nu_{A}^{2}}{2}\right\}$. Again, similar to Theorems 1 and 2 , we identify and compare all the KKT points of the function to find the optimal solution. It turns out that the function is maximized at $\left(e_{B}^{(1)}, r_{B}^{(1)}\right)=(0,0)$ with value $r_{A}^{(1)}\left(1-\nu_{A}\right)$, under an additional condition: $0<\nu_{A}<\frac{1}{3}$.

In conclusion, the optimal value of $\mathbb{E}\left[\pi_{B}^{(1)}\left(r_{A}^{(1)}, a_{B}^{(1)}\right)\right]$ is attained as $\mathbb{E}\left[\pi_{B}^{(1) *}\left(r_{A}^{(1)}\right)\right]=$ $r_{A}^{(1)}\left(1-\nu_{A}\right)$ at $\left(e_{B}^{(1) *}, r_{B}^{(1) *}\right)=(0,0)$, under the given conditions. So Theorem 3 is true. 
At last, we consider Alice in round 1. We have already proved in Theorem 1, Theorem 2 and Theorem 3 that $e_{B}^{(1) *}=e_{A}^{(2) *}=e_{B}^{(2) *}=r_{B}^{(1) *}=0$ and $f_{A}^{(2) *}=\frac{1}{2}\left(r_{B}^{(1)}\right)^{2}=0$ no matter what effort Alice exerts and no matter what report she makes in round 1 . In this situation $f_{A}^{(2) *}=0$ and we get the expression of $f_{A}^{(1)}+\delta f_{A}^{(2) *}=\mathbb{E}\left[\pi_{A}^{(1)}\left(r_{B}^{(0)}, a_{A}^{(1)}\right)\right]$, where $a_{A}^{(1)}=\left(e_{A}^{(1)}, r_{A}^{(1)}\right)$, as:

$$
\begin{aligned}
f_{A}^{(1)}+\delta f_{A}^{(2) *}=- & \alpha\left(e_{A}^{(1)}\right)^{2}+2 \nu_{A} e_{A}^{(1)}\left(r_{A}^{(1)}-r_{B}^{(0)}\right) \\
& +2\left(\left(r_{B}^{(0)}\right)^{2}-\left(r_{A}^{(1)}\right)^{2}\right)
\end{aligned}
$$

We now move to Alice's optimal actions in the first round. We find that Alice will exert low effort and report as 0 in round 1 regardless of the initial market estimate.

Theorem 4. If high efforts bring net costs and $\alpha>\max \left\{\frac{\left(1-\nu_{A}\right)^{2}}{2}, \frac{\nu_{A}^{2}}{2}\right\}$, then Alice's optimal action set in round 1 is $\left(e_{A}^{(1) *}, r_{A}^{(1) *}\right)=(0,0)$, and her expected total payoff score from this round to the final round is $\mathbb{E}\left[\pi_{A}^{(1) *}\left(r_{B}^{(0)}\right)\right]=$ $2\left(r_{B}^{(0)}\right)^{2}$

Proof. Similar to previous theorems, we can show that the function $f_{A}^{(1)}+\delta$. $f_{A}^{(2) *}$ is concave. We can also find its KKT points. By comparing the function values of the feasible KKT points, we immediately conclude that the optimal value of $f_{A}^{(1)}+\delta \cdot f_{A}^{(2) *}$ is achieved as $2\left(r_{B}^{(0)}\right)^{2}$ when $\left(e_{A}^{(1) *}, r_{A}^{(1) *}\right)=(0,0)$. Details are omitted. So Theorem 4 is true.

Summarizing results in Theorems 1, 2, 3, and 4, we infer the set of equilibrium strategies and payoffs for both agents of 2 rounds in table 3 (under the conditions given in the theorems).

\begin{tabular}{|c|c|c|c|}
\hline Round $i$ & Player $j$ & $a_{j}^{(i) *}=\left(e_{j}^{(i) *}, r_{j}^{(i) *}\right)$ & $\mathbb{E}\left[\pi_{j}^{(i) *},.\right]$ \\
\hline 1 & $A$ & $a_{A}^{(1) *}=(0,0)$ & $2\left(r_{B}^{(0)}\right)^{2}$ \\
\hline 1 & $B$ & $a_{B}^{(1) *}=(0,0)$ & 0 \\
\hline 2 & $A$ & $a_{A}^{(2) *}=(0,0)$ & 0 \\
\hline 2 & $B$ & $a_{B}^{(2) *}=(0,0)$ & 0 \\
\hline
\end{tabular}

Table 3: Equilibrium strategies and payoffs in case 1

We can immediately conclude that if $\alpha>\max \left\{\frac{\left(1-\nu_{A}\right)^{2}}{2}, \frac{\nu_{A}^{2}}{2}\right\}$ and $0<$ $\nu_{A}<\frac{1}{3}$, then not only will all of the agents' reports be 0 , but so will all 
of their effort values. This suggests that even when agents are strategic and external incentives exist, the proposed market scoring rule is incentive compatible regardless of the initial market estimate, so long as we set a proper range for $\alpha$ and $\nu_{A}$. In the next section we derive the symmetric result when the cost of high effort is negative.

\subsection{Case 2: High efforts bring net rewards to agents}

In case 2 we assume that two agents' efforts together decide the outcome of $E$ and the ultimate value of security $F$ but high efforts will bring negative net costs (equivalent to positive net rewards) to the agents who exert them. We further assume the payoff function of the exerted effort is $\rho_{e}(e)=\alpha e^{2}(\alpha>0)$ :

$$
\begin{aligned}
& \rho_{e}\left(e_{A}^{(i)}\right)=\alpha\left(e_{A}^{(i)}\right)^{2}, \\
& \rho_{e}\left(e_{B}^{(i)}\right)=\alpha\left(e_{B}^{(i)}\right)^{2} .
\end{aligned}
$$

In order to compare case 2 with case 1 , we set the same range for $\alpha$ as $\alpha>\max \left\{\frac{\left(1-\nu_{A}\right)^{2}}{2}, \frac{\nu_{A}^{2}}{2}\right\}$. In this section we set Alice's impact on deciding the likelihood of $E$ as $\nu_{A} \in(0,1)$ and have the same definition of Alice's (Bob's) belief on the likelihood of E's final occurring held in each round as that of case 1 . Here we continue using the quadratic scoring rule in the prediction market. We also note that, since proofs for case 2 are similar to that of case 1 , some technical details will be omitted in this subsection.

In case 2 we still have $I_{A}^{(i)}=r_{B}^{(i-1)}$ and $I_{B}^{(i)}=r_{A}^{(i)}$ as in case 1. After inserting the reward functions of exerted efforts (19), the belief on the likelihood of E's occurring (7) perceived by agents, and the reward functions in the prediction market (11) into the maximization equations (8) for Alice and $B$ ob separately, we get the following maximization equations when $T=2$. 
For agent Alice:

$$
\begin{aligned}
\mathbb{E}\left[\pi_{A}^{(1) *}\left(r_{B}^{(0)}\right)\right]= & \max _{\left(e_{A}^{(1)}, r_{A}^{(1)}\right)}\left\{\delta \mathbb{E}\left[\pi_{A}^{(2) *}\left(\tilde{r}_{B}^{(1)}\right)\right]+\alpha\left(e_{A}^{(1)}\right)^{2}\right. \\
& +2\left(\nu_{A}\left(e_{A}^{(1)}+\tilde{e}_{A}^{(2)}\right)+\left(1-\nu_{A}\right) \sum_{n=1}^{2} \tilde{e}_{B}^{(n)}\right)\left(r_{A}^{(1)}-r_{B}^{(0)}\right) \\
& \left.+2\left(\left(r_{B}^{(0)}\right)^{2}-\left(r_{A}^{(1)}\right)^{2}\right)\right\} \\
\mathbb{E}\left[\pi_{A}^{(2) *}\left(r_{B}^{(1)}\right)\right]= & \max _{\left(e_{A}^{(2)}, r_{A}^{(2)}\right)}\left\{\alpha\left(e_{A}^{(2)}\right)^{2}\right. \\
& +2\left(r_{B}^{(1)}+\nu_{A} e_{A}^{(2)}+\left(1-\nu_{A}\right) \tilde{e}_{B}^{(2)}\right)\left(r_{A}^{(2)}-r_{B}^{(1)}\right) \\
& \left.+2\left(\left(r_{B}^{(1)}\right)^{2}-\left(r_{A}^{(2)}\right)^{2}\right)\right\}
\end{aligned}
$$

For agent Bob:

$$
\begin{aligned}
& \mathbb{E}\left[\pi_{B}^{(1) *}\left(r_{A}^{(1)}\right)\right]=\max _{\left(e_{B}^{(1)}, r_{B}^{(1)}\right)}\left\{\delta \mathbb{E}\left[\pi_{B}^{(2) *}\left(\tilde{r}_{A}^{(2)}\right)\right]+\alpha\left(e_{B}^{(1)}\right)^{2}\right. \\
& +2\left(r_{A}^{(1)}+\nu_{A} \tilde{e}_{A}^{(2)}+\left(1-\nu_{A}\right)\left(e_{B}^{(1)}+\tilde{e}_{B}^{(2)}-0.5\right)\right) \\
& \left.\cdot\left(r_{B}^{(1)}-r_{A}^{(1)}\right)+2\left(\left(r_{A}^{(1)}\right)^{2}-\left(r_{B}^{(1)}\right)^{2}\right)\right\}, \\
& \mathbb{E}\left[\pi_{B}^{(2) *}\left(r_{A}^{(2)}\right)\right]=\max _{\left(e_{B}^{(2)}, r_{B}^{(2)}\right)}\left\{\alpha\left(e_{B}^{(2)}\right)^{2}\right. \\
& +2\left(2 r_{A}^{(2)}+\left(1-\nu_{A}\right)\left(e_{B}^{(2)}-0.5\right)\right)\left(r_{B}^{(2)}-r_{A}^{(2)}\right) \\
& \left.+2\left(\left(r_{A}^{(2)}\right)^{2}-\left(r_{B}^{(2)}\right)^{2}\right)\right\} .
\end{aligned}
$$

Next, similar to case 1, we identify all KKT points of the function and then find the optimal solutions.

In round 2, we consider Bob's function of expected payoff scores $f_{B}^{(2)}=$ $\mathbb{E}\left[\pi_{B}^{(2)}\left(r_{A}^{(2)}, a_{B}^{(2)}\right)\right]$ for action $a_{B}^{(2)}=\left(e_{B}^{(2)}, r_{B}^{(2)}\right)$ :

$$
\begin{aligned}
f_{B}^{(2)}= & \alpha\left(e_{B}^{(2)}\right)^{2}+2\left(2 r_{A}^{(2)}+\left(1-\nu_{A}\right)\left(e_{B}^{(2)}-0.5\right)\right)\left(r_{B}^{(2)}-r_{A}^{(2)}\right) \\
& +2\left(\left(r_{A}^{(2)}\right)^{2}-\left(r_{B}^{(2)}\right)^{2}\right) .
\end{aligned}
$$

Lemma 5. If $\alpha>\max \left\{\frac{\left(1-\nu_{A}\right)^{2}}{2}, \frac{\nu_{A}^{2}}{2}\right\}$ and $r_{A}^{(2)} \leqslant \frac{3+\nu_{A}}{4}$, then $\left(e_{B}^{(2) *}, r_{B}^{(2) *}\right)=$ $\left(1, r_{A}^{(2)}+\frac{1}{4}\left(1-\nu_{A}\right)\right)$ and $\mathbb{E}\left[\pi_{B}^{(2) *}\left(r_{A}^{(2)}\right)\right]=\alpha+\frac{1}{8}\left(1-\nu_{A}\right)^{2}$. 
Proof. Similar to Lemma 1, we first find the Hessian of function $f_{B}^{(2)}$ :

$$
\nabla^{2} f_{B}^{(2)}\left(e_{B}^{(2)}, r_{B}^{(2)}\right)=\left[\begin{array}{ll}
\frac{\partial^{2} f_{B}^{(2)}}{\partial\left(e_{B}^{(2)}\right)^{2}} & \frac{\partial^{2} f_{B}^{(2)}}{\partial e_{B}^{(2)} \partial r_{B}^{(2)}} \\
\frac{\partial^{2} f_{B}^{(2)}}{\partial r_{B}^{(2)} \partial e_{B}^{(2)}} & \frac{\partial^{2} f_{B}^{(2)}}{\partial\left(r_{B}^{(2)}\right)^{2}}
\end{array}\right]=\left[\begin{array}{lr}
2 \alpha & 2\left(1-\nu_{A}\right) \\
2\left(1-\nu_{A}\right) & -4
\end{array}\right]
$$

The first principle minor of $\nabla^{2} f_{B}^{(2)}\left(e_{B}^{(2)}, r_{B}^{(2)}\right)$ is positive for det $\left[\frac{\partial^{2} f_{B}^{(2)}}{\partial\left(e_{B}^{(2)}\right)^{2}}\right]=$ $2 \alpha>0$. the second principle minor is negative because:

$$
\operatorname{det}\left[\begin{array}{cc}
\frac{\partial^{2} f_{B}^{(2)}}{\partial\left(e_{B}^{(2)}\right)^{2}} & \frac{\partial^{2} f_{B}^{(2)}}{\partial e_{B}^{(2)} \partial r_{B}^{(2)}} \\
\frac{\partial^{2} f_{B}^{(2)}}{\partial r_{B}^{(2)} \partial e_{B}^{(2)}} & \frac{\partial^{2} f_{B}^{(2)}}{\partial\left(r_{B}^{(2)}\right)^{2}}
\end{array}\right]=-8 \alpha-4\left(1-\nu_{A}\right)^{2}<0 .
$$

We find that the Hessian of $f_{B}^{(2)}$ is an indefinite matrix so we need to find all KKT points and compare the corresponding values of $f_{B}^{(2)}$ for each point, since $f_{B}^{(2) *}$ must be attained in one of these KKT points. All (feasible) KKT points of $f_{B}^{(2)}$ and their corresponding $f_{B}^{(2)}$ function values are presented in Table 4. (Note: To simplify our analysis, unfeasible KKT points are not shown in tables in this subsection.) We find that if $r_{A}^{(2)} \leqslant \frac{3+\nu_{A}}{4}, f_{B}^{(2) *}$ is attained as $\alpha+\frac{1}{8}\left(1-\nu_{A}\right)^{2}$ when $\left(e_{B}^{(2)}, r_{B}^{(2)}\right)=\left(1, r_{A}^{(2)}+\frac{1}{4}\left(1-\nu_{A}\right)\right)$. So Lemma 5 is true.

Lemma 6. If $\alpha>\max \left\{\frac{\left(1-\nu_{A}\right)^{2}}{2}, \frac{\nu_{A}^{2}}{2}\right\}$ and $r_{A}^{(2)} \geqslant \frac{3+\nu_{A}}{4}$, then $\left(e_{B}^{(2) *}, r_{B}^{(2) *}\right)=$ $(1,1)$ and $\mathbb{E}\left[\pi_{B}^{(2) *}\left(r_{A}^{(2)}\right)\right]=-2\left(r_{A}^{(2)}\right)^{2}+\left(\nu_{A}+3\right) r_{A}^{(2)}+\alpha-\nu_{A}-1$.

Proof. Similar as the proof for Lemma 5, if $r_{A}^{(2)} \geqslant \frac{3+\nu_{A}}{4}$, by comparing the values of $f_{B}^{(2)}$ of feasible KKT points, we find that $f_{B}^{(2) *}$ is attained as $f_{B}^{(2) *}=-2\left(r_{A}^{(2)}\right)^{2}+\left(\nu_{A}+3\right) r_{A}^{(2)}+\alpha-\nu_{A}-1$ when $\left(e_{B}^{(2) *}, r_{B}^{(2) *}\right)=(1,1)$. Details are omitted. So Lemma 6 is true.

Now, we are ready to determine Bob's round 2 decisions: 


\begin{tabular}{|c|c|c|}
\hline$e_{B}^{(2)}$ & $r_{B}^{(2)}$ & $f_{B}^{(2)}$ \\
\hline 0 & $r_{A}^{(2)}-\frac{1}{4}\left(1-\nu_{A}\right)$ & $\frac{1}{8}\left(1-\nu_{A}\right)^{2}$ \\
\hline 1 & $r_{A}^{(2)}+\frac{1}{4}\left(1-\nu_{A}\right)$ & $\alpha+\frac{1}{8}\left(1-\nu_{A}\right)^{2}$ \\
\hline$\frac{r_{A}^{(2)}\left(1-\nu_{A}\right)}{\alpha}$ & 0 & $-2\left(r_{A}^{(2)}\right)^{2}+r_{A}^{(2)}\left(1-\nu_{A}\right)-\frac{\left(r_{A}^{(2)}\right)^{2}\left(1-\nu_{A}\right)^{2}}{\alpha}$ \\
\hline 0 & 0 & $-2\left(r_{A}^{(2)}\right)^{2}+\left(1-\nu_{A}\right) r_{A}^{(2)}$ \\
\hline 0 & 1 & $-2\left(r_{A}^{(2)}\right)^{2}+\left(5-\nu_{A}\right) r_{A}^{(2)}+\nu_{A}-3$ \\
\hline 1 & 0 & $-2\left(r_{A}^{(2)}\right)^{2}+\left(\nu_{A}-1\right) r_{A}^{(2)}+\alpha$ \\
\hline 1 & 1 & $-2\left(r_{A}^{(2)}\right)^{2}+\left(\nu_{A}+3\right) r_{A}^{(2)}+\alpha-\nu_{A}-1$ \\
\hline
\end{tabular}

Table 4: KKT points of $f_{B}^{(2)}$ in case 2

Theorem 5. If high efforts bring net rewards and $\alpha>\max \left\{\frac{\left(1-\nu_{A}\right)^{2}}{2}, \frac{\nu_{A}^{2}}{2}\right\}$, then

$$
\begin{gathered}
\left(e_{B}^{(2) *}, r_{B}^{(2) *}\right)= \begin{cases}\left(1, r_{A}^{(2)}+\frac{1}{4}\left(1-\nu_{A}\right)\right), & \text { if } 0 \leqslant r_{A}^{(2)} \leqslant \frac{3+\nu_{A}}{4} ; \\
(1,1), & \text { if } \frac{3+\nu_{A}}{4} \leqslant r_{A}^{(2)} \leqslant 1 .\end{cases} \\
f_{B}^{(2) *}= \begin{cases}\alpha+\frac{1}{8}\left(1-\nu_{A}\right)^{2}, & \text { if } 0 \leqslant r_{A}^{(2)} \leqslant \frac{3+\nu_{A}}{4} ; \\
-2\left(r_{A}^{(2)}\right)^{2}+\left(\nu_{A}+3\right) r_{A}^{(2)}+\alpha-\nu_{A}-1, & \text { if } \frac{3+\nu_{A}}{4} \leqslant r_{A}^{(2)} \leqslant 1 .\end{cases}
\end{gathered}
$$

Proof. With Lemma 5 and Lemma 6, Theorem 5 is true.

Then we consider Alice in round $i=2$ when Bob's future effort is $\tilde{e}_{B}^{(2)}$. We know from Theorem 5 that $\tilde{e}_{B}^{(2)}=e_{B}^{(2) *}=1$. After inserting $\tilde{e}_{B}^{(2)}=1$ into $f_{A}^{(2)}=\mathbb{E}\left[\pi_{A}^{(2)}, r_{B}^{(1)}, a_{A}^{(2)}\right]$, we get:

$$
\begin{aligned}
f_{A}^{(2)}=\alpha\left(e_{A}^{(2)}\right)^{2} & +2\left(r_{B}^{(1)}+\nu_{A} \cdot e_{A}^{(2)}+1-\nu_{A}\right)\left(r_{A}^{(2)}-r_{B}^{(1)}\right) \\
& +2\left(\left(r_{B}^{(1)}\right)^{2}-2\left(r_{A}^{(2)}\right)^{2}\right) .
\end{aligned}
$$

For Alice's optimal actions in round 2, we find that Alice will exert high effort and report as 1 in round 2 no matter what $B$ ob reports in round 1.

Theorem 6. If high efforts bring net rewards and $\alpha>\max \left\{\frac{\left(1-\nu_{A}\right)^{2}}{2}, \frac{\nu_{A}^{2}}{2}\right\}$, Alice's optimal action set in round 2 is $\left(e_{A}^{(2) *}, r_{A}^{(2) *}\right)=\left(1, \frac{1}{2}\left(r_{B}^{(1)}+1\right)\right)$, and the optimal expected payoff score of round 2 is $\mathbb{E}\left[\pi_{A}^{(2) *}\left(r_{B}^{(1)}\right)\right]=\alpha+\frac{1}{2}\left(r_{B}^{(1)}\right)^{2}+$ $\frac{1}{2}-r_{B}^{(1)}$. 


\begin{tabular}{|c|c|c|}
\hline$e_{A}^{(2)}$ & $r_{A}^{(2)}$ & $f_{A}^{(2)}$ \\
\hline 0 & $\frac{1}{2} r_{B}^{(1)}+\frac{1}{2}\left(1-\nu_{A}\right)$ & $\frac{1}{2}\left(r_{B}^{(1)}\right)^{2}+\frac{1}{2}\left(1-\nu_{A}\right)^{2}-r_{B}^{(1)}\left(1-\nu_{A}\right)$ \\
\hline 1 & $\frac{1}{2}\left(r_{B}^{(1)}+1\right)$ & $\alpha+\frac{1}{2}\left(r_{B}^{(1)}\right)^{2}+\frac{1}{2}-r_{B}^{(1)}$ \\
\hline$\frac{\nu_{A} r_{B}^{(1)}}{\alpha}$ & 0 & $-2 r_{B}^{(1)}\left(1-\nu_{A}\right)-\frac{\left(\nu_{A} r_{B}^{(1)}\right)^{2}}{\alpha}$ \\
\hline 0 & 0 & $-2 r_{B}^{(1)}\left(1-\nu_{A}\right)$ \\
\hline 0 & 1 & $-2 \nu_{A}\left(1-r_{B}^{(1)}\right)$ \\
\hline 1 & 0 & $-2 r_{B}^{(1)}+\alpha$ \\
\hline 1 & 1 & $\alpha$ \\
\hline
\end{tabular}

Table 5: KKT points of $f_{A}^{(2)}$ in case 2

Proof. To find the value of $f_{A}^{(2) *}$, we find all the KKT points of $f_{A}^{(2)}$ and the corresponding function values (See Table 5). By routine calculations, we find that $f_{A}^{(2)}$ is maximized as $f_{A}^{(2) *}=\alpha+\frac{1}{2}\left(r_{B}^{(1)}\right)^{2}+\frac{1}{2}-r_{B}^{(1)}$ when $\left(e_{A}^{(2)}, r_{A}^{(2)}\right)=\left(1, \frac{1}{2}\left(r_{B}^{(1)}+1\right)\right)$. So Theorem 6 is true.

Now, we consider Bob in round 1. From Theorem 5 and Theorem 6 we know that $e_{A}^{(2) *}=e_{B}^{(2) *}=1$. Knowing $\tilde{e}_{A}^{(2)}=\tilde{e}_{B}^{(2)}=1$, we get the expression of $f_{B}^{(1)}$ as:

$$
\begin{aligned}
f_{B}^{(1)}=\alpha \cdot\left(e_{B}^{(1)}\right)^{2} & +2\left(r_{A}^{(1)}+\nu_{A}+\left(1-\nu_{A}\right)\left(e_{B}^{(1)}+0.5\right)\right)\left(r_{B}^{(1)}-r_{A}^{(1)}\right) \\
& +2\left(\left(r_{A}^{(1)}\right)^{2}-2\left(r_{B}^{(1)}\right)^{2}\right) .
\end{aligned}
$$

Next, we need three lemmas for the maximum value of $\mathbb{E}\left[\pi_{B}^{(1)}\left(r_{A}^{(2)}, a_{B}^{(1)}\right)\right]=$ $f_{B}^{(1)}+\delta f_{B}^{(2) *}$, where $a_{B}^{(1)}=\left(e_{B}^{(1)}, r_{B}^{(1)}\right)$.

Lemma 7. If $\alpha>\max \left\{\frac{\left(1-\nu_{A}\right)^{2}}{2}, \frac{\nu_{A}^{2}}{2}\right\}$, then

$$
\begin{gathered}
\left(e_{B}^{(2) *}, r_{B}^{(2) *}\right)= \begin{cases}\left(1, r_{A}^{(2)}+\frac{1}{4}\left(1-\nu_{A}\right)\right), & \text { if } 0 \leqslant r_{B}^{(1)} \leqslant \frac{1}{2}\left(1+\nu_{A}\right) ; \\
(1,1), & \text { if } \frac{1}{2}\left(1+\nu_{A}\right) \leqslant r_{B}^{(1)} \leqslant 1 .\end{cases} \\
f_{B}^{(2) *}=\left\{\begin{array}{lr}
\alpha+\frac{1}{8}\left(\nu_{A}-1\right)^{2}, & \text { if } 0 \leqslant r_{B}^{(1)} \leqslant \frac{1}{2}\left(1+\nu_{A}\right) ; \\
-\frac{1}{2}\left(r_{B}^{(1)}\right)^{2}+\frac{1}{2} r_{B}^{(1)}+\frac{1}{2} r_{B}^{(1)} \nu_{A}-\frac{1}{2} \nu_{A}+\alpha, & \text { if } \frac{1}{2}\left(1+\nu_{A}\right) \leqslant r_{B}^{(1)} \leqslant 1 .
\end{array}\right.
\end{gathered}
$$

Proof. By Theorem 6, we know that $r_{A}^{(2) *}=\frac{1}{2}\left(r_{B}^{(1)}+1\right)$. Together with the statement of Theorem 5, we infer Lemma 7. 
Lemma 8. If $\alpha>\max \left\{\frac{\left(1-\nu_{A}\right)^{2}}{2}, \frac{\nu_{A}^{2}}{2}\right\}$ and $r_{A}^{(1)} \leqslant \frac{\nu_{A}+1}{2}$, then $\left(e_{B}^{(1) *}, r_{B}^{(1) *}\right)=$ $\left(1, \frac{1}{2} r_{A}^{(1)}+\frac{1}{4}\left(3-\nu_{A}\right)\right)$ and

$$
\begin{aligned}
\mathbb{E}\left[\pi_{B}^{(1) *}\left(r_{A}^{(1)}\right)\right]= & \frac{1}{2} r_{A}^{(1)}\left(r_{A}^{(1)}-3+\nu_{A}\right)+\alpha+\frac{1}{8} \nu_{A}^{2} \\
& -\frac{3}{4} \nu_{A}+\frac{9}{8}+\delta\left(\alpha+\frac{1}{8}\left(1-\nu_{A}\right)^{2}\right) .
\end{aligned}
$$

Proof. From Lemma 7 we know that $f_{B}^{(2) *}$ is attained as $f_{B}^{(2) *}=\alpha+\frac{1}{8}\left(1-\nu_{A}\right)^{2}$ when $r_{B}^{(1)} \leqslant \frac{1}{2}\left(1+\nu_{A}\right)$; and attained as $f_{B}^{(2) *}=-\frac{1}{2}\left(r_{B}^{(1)}\right)^{2}+\frac{1}{2} r_{B}^{(1)}+\frac{1}{2} r_{B}^{(1)} \nu_{A}-$ $\frac{1}{2} \nu_{A}+\alpha$ when $r_{B}^{(1)} \geqslant \frac{1}{2}\left(1+\nu_{A}\right)$.

We first discuss the situation where $f_{B}^{(2) *}$ is a constant as $f_{B}^{(2) *}=\alpha+$ $\frac{1}{8}\left(1-\nu_{A}\right)^{2}$ when $r_{B}^{(1)} \leqslant \frac{1}{2}\left(1+\nu_{A}\right)$. We first find all the KKT points of $f_{B}^{(1)}+\delta \cdot f_{B}^{(2) *}$. We find that when $r_{A}^{(1)} \leqslant \frac{1+\nu_{A}}{2}, f_{B}^{(1)}+\delta \cdot f_{B}^{(2) *}$ is maximized as $\frac{1}{2} r_{A}^{(1)}\left(r_{A}^{(1)}-3+\nu_{A}\right)+\alpha+\frac{1}{8} \nu_{A}^{(2)}-\frac{3}{4} \nu_{A}+\frac{9}{8}+\delta\left(\alpha+\frac{1}{8}\left(\nu_{A}-1\right)^{2}\right)$ at $\left(e_{B}^{(1)}, r_{B}^{(1)}\right)=$ $\left(1, \frac{1}{2} r_{A}^{(1)}+\frac{1}{4}\left(3-\nu_{A}\right)\right)$.

Second, we discuss the situation where $f_{B}^{(2) *}$ is a function of $r_{B}^{(1)}$ as $f_{B}^{(2) *}=$ $-\frac{1}{2}\left(r_{B}^{(1)}\right)^{2}+\frac{1}{2} r_{B}^{(1)}+\frac{1}{2} r_{B}^{(1)} \nu_{A}-\frac{1}{2} \nu_{A}+\alpha$, if $r_{B}^{(1)} \geqslant \frac{1}{2}\left(1+\nu_{A}\right)$. Using the same method, we find that $f_{B}^{(1)}+\delta \cdot f_{B}^{(2) *}$ is maximized as $\left(1-\nu_{A}\right)\left(1-r_{A}^{(1)}\right)+\alpha(1+\delta)$ at $\left(e_{B}^{(1)}, r_{B}^{(1)}\right)=(1,1)$.

By routine calculations, we know that the first expression of $f_{B}^{(1)}+\delta$. $f_{B}^{(2) *}$ is larger than the value of the second expression. So $\mathbb{E}\left[\pi_{B}^{(1) *}\left(r_{A}^{(1)}\right)\right]$ is achieved as $\frac{1}{2} r_{A}^{(1)}\left(r_{A}^{(1)}-3+\nu_{A}\right)+\alpha+\frac{1}{8} \nu_{A}^{2}-\frac{3}{4} \nu_{A}+\frac{9}{8}+\delta\left(\alpha+\frac{1}{8}\left(\nu_{A}-1\right)^{2}\right)$ at $\left(e_{B}^{(1) *}, r_{B}^{(1) *}\right)=\left(1, \frac{1}{2} r_{A}^{(1)}+\frac{1}{4}\left(3-\nu_{A}\right)\right)$. So Lemma 8 is true.

Lemma 9. If $\alpha>\max \left\{\frac{\left(1-\nu_{A}\right)^{2}}{2}, \frac{\nu_{A}^{2}}{2}\right\}$ and $r_{A}^{(1)} \geqslant \frac{\nu_{A}+1}{2}$, then $\left(e_{B}^{(1) *}, r_{B}^{(1) *}\right)=$ $(1,1)$ and

$$
\mathbb{E}\left[\pi_{B}^{(1) *}\left(r_{A}^{(2)}\right)\right]=\left(1-\nu_{A}\right)\left(1-r_{A}^{(1)}\right)+\alpha(1+\delta)+\delta \nu_{A}\left(\frac{1}{2}-\nu_{A}\right)
$$

Proof. The proof is similar to that of Lemma 8. Details are omitted.

Given the two cases for Bob's equilibrium decisions in round 1, we may now determine $B$ ob's round 1 decisions:

Theorem 7. If high efforts bring net rewards and $\alpha>\max \left\{\frac{\left(1-\nu_{A}\right)^{2}}{2}, \frac{\nu_{A}^{2}}{2}\right\}$, then

$$
\left(e_{B}^{(1) *}, r_{B}^{(1) *}\right)= \begin{cases}\left(1, \frac{1}{2} r_{A}^{(1)}+\frac{1}{4}\left(3-\nu_{A}\right)\right), & \text { if } 0 \leqslant r_{A}^{(1)} \leqslant \frac{\nu_{A}+1}{2} \\ (1,1), & \text { if } \frac{\nu_{A}+1}{2} \leqslant r_{A}^{(1)} \leqslant 1\end{cases}
$$




$$
\mathbb{E}\left[\pi_{B}^{(1) *}\left(r_{A}^{(1)}\right)\right]= \begin{cases}\frac{1}{2} r_{A}^{(1)}\left(r_{A}^{(1)}-3+\nu_{A}\right)+\alpha+\frac{1}{8} \nu_{A}, & \\ -\frac{3}{4} \nu_{A}+\frac{9}{8}+\delta\left(\alpha+\frac{1}{8}\left(1-\nu_{A}\right)^{2}\right), & \text { if } 0 \leqslant r_{A}^{(1)} \leqslant \frac{\nu_{A}+1}{2} ; \\ \left(1-\nu_{A}\right)\left(1-r_{A}^{(1)}\right)+\alpha & \\ +\delta\left(\alpha+\nu_{A}\left(\frac{1}{2}-\nu_{A}\right)\right), & \text { if } \frac{\nu_{A}+1}{2} \leqslant r_{A}^{(1)} \leqslant 1 .\end{cases}
$$

Proof. With Lemma 8 and Lemma 9, Theorem 7 is true.

Finally, we consider Alice in round 1 . We have already proved in Theorem 5, Theorem 6 and Theorem 7 that $e_{B}^{(1) *}=e_{A}^{(2) *}=e_{B}^{(2) *}=1$ and $\mathbb{E}\left[\pi_{A}^{(2) *}\left(r_{B}^{(1)}\right)\right]=\alpha+\frac{1}{2}\left(r_{B}^{(1)}\right)^{2}+\frac{1}{2}-r_{B}^{(1)}$. After inserting $\tilde{e}_{B}^{(1)}=\tilde{e}_{A}^{(2)}=\tilde{e}_{B}^{(2)}=1$ and $\mathbb{E}\left[\pi_{A}^{(2) *}\left(\tilde{r}_{B}^{(1)}\right)\right]$ into $\mathbb{E}\left[\pi_{A}^{(1)}\left(r_{B}^{(0)}, a_{A}^{(1)}\right)\right]$, we obtain

$$
\begin{aligned}
\mathbb{E} & {\left[\pi_{A}^{(1)}\left(r_{B}^{(1)}, a_{A}^{(2)}\right)\right]=f_{A}^{(1)}+\delta f_{A}^{(2) *} } \\
= & \delta\left(\alpha+\frac{1}{2}(1)^{2}+\frac{1}{2}-1\right)+\alpha\left(e_{A}^{(1)}\right)^{2} \\
& \quad+2\left(\nu_{A} e_{A}^{(1)}+2-\nu_{A}\right)\left(r_{A}^{(1)}-r_{B}^{(0)}\right)+2\left(\left(r_{B}^{(0)}\right)^{2}-2\left(r_{A}^{(1)}\right)^{2}\right) \\
= & \alpha\left(\delta+\left(e_{A}^{(1)}\right)^{2}\right) \\
& \quad+2\left(\nu_{A} e_{A}^{(1)}+2-\nu_{A}\right)\left(r_{A}^{(1)}-r_{B}^{(0)}\right)+2\left(\left(r_{B}^{(0)}\right)^{2}-\left(r_{A}^{(1)}\right)^{2}\right)
\end{aligned}
$$

We now move to Alice's optimal actions in the first round. We find that Alice will exert high effort and report as 1 in round 1 no matter what initial market estimate is.

Theorem 8. If $\alpha>\max \left\{\frac{\left(1-\nu_{A}\right)^{2}}{2}, \frac{\nu_{A}^{2}}{2}\right\}$, then Alice's optimal action set in round 1 is $\left(e_{A}^{(1) *}, r_{A}^{(1) *}\right)=(1,1)$, and her expected total payoff score from this round to the final round is $\mathbb{E}\left[\pi_{A}^{(1) *}\left(r_{B}^{(0)}\right)\right]=2\left(r_{B}^{(0)}-1\right)^{2}+(1+\delta) \alpha$.

Proof. Similar to previous theorems, we first identify all the KKT points of $f_{A}^{(1)}+\delta f_{A}^{(2) *}$. We then find that the optimal value of $f_{A}^{(1)}+\delta \cdot f_{A}^{(2) *}$ is achieved as $2\left(r_{B}^{(0)}-1\right)^{2}+(1+\delta) \alpha$ when $\left(e_{A}^{(1) *}, r_{A}^{(1) *}\right)=(1,1)$. Details are omitted. So Theorem 8 is true.

Summarizing results in Theorem 8, Theorem 7, Theorem 6, and Theorem 5 , we infer the set of optimal equilibrium strategies $a_{j}^{(i) *}=\left(e_{j}^{(i) *}, r_{j}^{(i) *}\right)$ and their corresponding payoffs for both agents of 2 rounds in table 6 (under the conditions specified in the theorems): 


\begin{tabular}{|c|c|c|c|}
\hline Round, $i$ & Player, $j$ & $a_{j}^{(i) *}=\left(e_{j}^{(i) *}, r_{j}^{(i) *}\right)$ & $E\left[\pi_{j}^{(i) *}(\cdot)\right]$ \\
\hline 1 & $A$ & $a_{A}^{(1) *}=(1,1)$ & $2\left(r_{B}^{(0)}-1\right)^{2}+(1+\delta) \alpha$ \\
\hline 1 & $B$ & $a_{B}^{(1) *}=(1,1)$ & $\alpha(1+\delta)+\delta \nu_{A}\left(\frac{1}{2}-\nu_{A}\right)$ \\
\hline 2 & $A$ & $a_{A}^{(2) *}=(1,1)$ & $\alpha$ \\
\hline 2 & $B$ & $a_{B}^{(2) *}=(1,1)$ & $\alpha$ \\
\hline
\end{tabular}

Table 6: Equilibrium strategies and payoffs in case 2

We can immediately conclude that if $\alpha>\max \left\{\frac{\left(1-\nu_{A}\right)^{2}}{2}, \frac{\nu_{A}^{2}}{2}\right\}$, then not only will all of the agents' reports be 1 , but so will all of their effort values. The results in sections 4.2 and 4.3 generalize to the 4 round setting, but it is still unclear if they generalize for any value of $T$.

We carried out numerical simulations, via simulated annealing, for $T>$ 4 and found that starting with random values of $r_{j}^{(i)}$ and $e_{j}^{(i)}$, for player $j \in\{A, B\}$, over all rounds, the final Pareto dominant solution was either one with all players reporting truthfully and exerting high effort (referred to as the truthful solution), or mostly exerting high effort and being truthful with some deviation in effort and reports, called the mixed solution. The interesting aspect of our numerical simulations, is that for the solutions we found, the truthful solution always had a higher payoff for Alice than the mixed solution, and Bob had a higher payoff in the mixed solution than in the truthful solution. In addition, the values of the payoff functions tended to differ only in the hundredths or thousandths place of the reward functions values across the two solutions. Given our findings, we conclude that the results of the numerical simulation are inconclusive. We say the results are inconclusive because we started with a random set of actions for all players across all rounds, and the Pareto dominant solution we find may be infeasible as Alice is the first mover in the game. The first mover, Alice may take actions to avoid ever getting to the initial state of the game (where we started the simulation), as she is rational, she would receive a higher payoff in the truthful solution than the mixed solution and will take actions to avoid the initial state of the simulation. In order for a simulation to make sense, it must take into account Alice's first-mover advantage.

\subsection{Case 3: External incentives do not exist}

In case 3 we assume that two agents' efforts together decide the outcome of $E$ and the ultimate value of security $F$ but their efforts will bring no payoffs to the agents who exert them $\left(\rho_{e}(e)=0\right)$. Previous work has shown 
that when external incentives do not exist outside of the prediction market, non-myopic agents have incentives to bluff in the prediction market (Chen et al., 2010; Dimitrov and Sami, 2008). We show in case 3 that this result also applies for our model. We assume $\nu_{A}=\frac{1}{2}$ (Alice and $B$ ob have the same impact on deciding the occurring probability of $E$ ) and $r_{B}^{(0)}=\frac{3}{4}$ (the initial market estimate is set to be $\frac{3}{4}$ ). Under the above assumptions we then have Alice and Bob's Bellman equations in the 2-round setting as: For agent Alice (with $r_{B}^{(0)}=\frac{3}{4}$ ):

$$
\begin{aligned}
\mathbb{E}\left[\pi_{A}^{(1) *}\left(r_{B}^{(0)}\right)\right]= & \max _{\left(e_{A}^{(1)}, r_{A}^{(1)}\right)}\left\{\delta \mathbb{E}\left[\pi_{A}^{(2) *}\left(\tilde{r}_{B}^{(1)}\right)\right]\right. \\
& +2\left(\nu_{A}\left(e_{A}^{(1)}+\tilde{e}_{A}^{(2)}\right)+\left(1-\nu_{A}\right)\left(\tilde{e}_{B}^{(1)}+\tilde{e}_{B}^{(2)}\right)\right)\left(r_{A}^{(1)}-r_{B}^{(0)}\right) \\
& \left.+2\left(\left(r_{B}^{(0)}\right)^{2}-\left(r_{A}^{(1)}\right)^{2}\right)\right\} \\
\mathbb{E}\left[\pi_{A}^{(2) *}\left(r_{B}^{(1)}\right)\right]= & \max _{\left(e_{A}^{(2)}, r_{A}^{(2)}\right)}\left\{2\left(r_{B}^{(1)}+\nu_{A} \cdot \tilde{e}_{A}^{(2)}+\left(1-\nu_{A}\right) \tilde{e}_{B}^{(2)}\right)\right. \\
& \left.\cdot\left(r_{A}^{(2)}-r_{B}^{(1)}\right)+2\left(\left(r_{B}^{(1)}\right)^{2}-\left(r_{A}^{(2)}\right)^{2}\right)\right\}
\end{aligned}
$$

For agent Bob:

$$
\begin{aligned}
& \mathbb{E}\left[\pi_{B}^{(1) *}\left(r_{A}^{(1)}\right)\right]= \max _{\left(e_{B}^{(1)}, r_{B}^{(1)}\right)}\left\{\delta \mathbb{E}\left[\pi_{B}^{(2) *}\left(\tilde{r}_{A}^{(2)}\right)\right]\right. \\
&+ 2\left(r_{A}^{(1)}+\nu_{A} \cdot \tilde{e}_{A}^{(2)}+\left(1-\nu_{A}\right)\left(e_{B}^{(1)}+\tilde{e}_{B}^{(2)}-0.5\right)\right) \\
&\left.\cdot\left(r_{B}^{(1)}-r_{A}^{(1)}\right)+\left(\left(r_{A}^{(1)}\right)^{2}-\left(r_{B}^{(1)}\right)^{2}\right)\right\}, \\
& \mathbb{E}\left[\pi_{B}^{(2) *}\left(r_{A}^{(2)}\right)\right]=\max _{\left(e_{B}^{(2)}, r_{B}^{(2)}\right)}\left\{2\left(r_{A}^{(2)}+\left(1-\nu_{A}\right)\left(e_{B}^{(2)}-0.5\right)\right)\right. \\
&\left.\cdot\left(r_{B}^{(2)}-r_{A}^{(2)}\right)+2\left(\left(r_{A}^{(2)}\right)^{2}-\left(r_{B}^{(2)}\right)^{2}\right)\right\} .
\end{aligned}
$$

Using backwards induction as has been explained in detail for case 1 and case 2 , we infer the set of equilibrium strategies $\left(e_{j}^{(i) *}, r_{j}^{(i) *}\right)$ and their corresponding payoffs $E\left[\pi_{j}^{(i) *}(\cdot)\right]$ for both agents of 2 rounds in Table 7, under the initial condition $r_{B}^{(0)}=\frac{3}{4}$. Technical details are omitted.

So in case 3 where external incentives do not exist, we observe directly from the optimal actions that agents do not necessarily exert high efforts to $E$ and they also do not report truthfully in the prediction market. 


\begin{tabular}{|c|c|c|c|}
\hline Round, $i$ & Player, $j$ & $a_{j}^{(i) *}=\left(e_{j}^{(i) *}, r_{j}^{(i) *}\right)$ & $E\left[\pi_{j}^{(i) *}(\cdot)\right]$ \\
\hline 1 & $A$ & $a_{A}^{(1) *}=\left(0, \frac{1}{2}\right)$ & $\frac{1}{8}$ \\
\hline 1 & $B$ & $a_{B}^{(1) *}=\left(1, \frac{7}{8}\right)$ & $\frac{1}{32}$ \\
\hline 2 & $A$ & $a_{A}^{(2) *}=\left(0, \frac{11}{16}\right)$ & $\frac{9}{128}+\frac{1}{8} \delta$ \\
\hline 2 & $B$ & $a_{B}^{(2) *}=\left(1, \frac{13}{16}\right)$ & $\frac{1}{32}(1+\delta)$ \\
\hline
\end{tabular}

Table 7: Equilibrium strategies in case 3

4.5. Case 4: High efforts bring net rewards to agents in a 4-round setting

In this case, similar to Case 2, we assume that high efforts will bring negative net costs (equivalent to positive net rewards) to the agents who exert them. We extend the number of rounds from 2 rounds to 4 rounds. In order to properly compare case 4 with the previous cases, we assume the payoff function of exerted effort to be $\rho_{e}(e)=\alpha \cdot e^{2}(\alpha>0)$ as we have assumed for case 2 in (19), and we set the same range for $\alpha$ as $\alpha>$ $\max \left\{\frac{\left(1-\nu_{A}\right)^{2}}{2}, \frac{\nu_{A}^{2}}{2}\right\}$. We set Alice's impact on deciding the likelihood of $E$ as $\nu_{A} \in(0,1)$ and have the same definition of Alice's (Bob's) belief on the likelihood of $E$ 's final occurring held in each round as that of case 1 . Here we continue using the quadratic scoring rule in the prediction market. In case 4 we still have $I_{A}^{(i)}=r_{B}^{(i-1)}$ and $I_{B}^{(i)}=r_{A}^{(i)}$ as in case 1 and case 2.

After inserting the reward functions of exerted efforts (19), the belief on the likelihood of E's occurring (7) perceived by agents, and the reward functions in the prediction market (11) into the maximization equations (8) for Alice and Bob separately, we get the following maximization equations when $T=4$ : 
For agent Alice:

$$
\begin{aligned}
\mathbb{E}\left[\pi_{A}^{(1) *}\left(r_{B}^{(0)}\right)\right]= & \max _{\left(e_{A}^{(1)}, r_{A}^{(1)}\right)}\left\{\delta \mathbb{E}\left[\pi_{A}^{(2) *}\left(\tilde{r}_{B}^{(1)}\right)\right]+\alpha\left(e_{A}^{(1)}\right)^{2}\right. \\
& +\left(\nu_{A}\left(e_{A}^{(1)}+\sum_{n=2}^{4} \tilde{e}_{A}^{(n)}\right)+\left(1-\nu_{A}\right) \sum_{n=1}^{4} \tilde{e}_{B}^{(n)}\right)\left(r_{A}^{(1)}-r_{B}^{(0)}\right) \\
& \left.+2\left(\left(r_{B}^{(0)}\right)^{2}-\left(r_{A}^{(1)}\right)^{2}\right)\right\} \\
\mathbb{E}\left[\pi_{A}^{(2) *}\left(r_{B}^{(1)}\right)\right]= & \max _{\left(e_{A}^{(2)}, r_{A}^{(2)}\right)}\left\{\delta \mathbb{E}\left[\pi_{A}^{(3) *}\left(\tilde{r}_{B}^{(2)}\right)\right]+\alpha\left(e_{A}^{(2)}\right)^{2}\right. \\
& +\left(r_{B}^{(1)}+\nu_{A}\left(e_{A}^{(2)}+\sum_{n=3}^{4} \tilde{e}_{A}^{(n)}\right)+\left(1-\nu_{A}\right) \sum_{n=2}^{4} \tilde{e}_{B}^{(n)}\right)\left(r_{A}^{(2)}-r_{B}^{(1)}\right) \\
& \left.+2\left(\left(r_{B}^{(1)}\right)^{2}-\left(r_{A}^{(2)}\right)^{2}\right)\right\} \\
\mathbb{E}\left[\pi_{A}^{(3) *}\left(r_{B}^{(2)}\right)\right]= & \max _{\left(e_{A}^{(3)}, r_{A}^{(3)}\right)}\left\{\delta \mathbb{E}\left[\pi_{A}^{(4) *}\left(\tilde{r}_{B}^{(3)}\right)\right]+\alpha\left(e_{A}^{(3)}\right)^{2}\right. \\
& +\left(2 r_{B}^{(2)}+\nu_{A}\left(e_{A}^{(3)}+\tilde{e}_{A}^{(4)}\right)+\left(1-\nu_{A}\right) \sum_{n=3}^{4} \tilde{e}_{B}^{(n)}\right)\left(r_{A}^{(3)}-r_{B}^{(2)}\right) \\
& \left.+2\left(\left(r_{B}^{(2)}\right)^{2}-\left(r_{A}^{(3)}\right)^{2}\right)\right\} \\
& \left.+2\left(\left(r_{B}^{(3)}\right)^{2}-\left(r_{A}^{(4)}\right)^{2}\right)\right\} \\
\mathbb{E}\left[\pi_{A}^{(4) *}\left(r_{B}^{(3)}\right)\right]= & \max _{\left(e_{A}^{(4)}, r_{A}^{(4)}\right)}\left\{\alpha\left(e_{A}^{(4)}\right)^{2}\right. \\
& +\left(3 r_{B}^{(3)}+\nu_{A} e_{A}^{(4)}+\left(1-\nu_{A}\right) \tilde{e}_{B}^{(4)}\right)\left(r_{A}^{(4)}-r_{B}^{(3)}\right) \\
& +28 \mathrm{c}) \\
& (28 \mathrm{~d}) \\
& (28) \\
& (2)
\end{aligned}
$$


For agent Bob:

$$
\begin{aligned}
\mathbb{E}\left[\pi_{B}^{(1) *}\left(r_{A}^{(1)}\right)\right]= & \max _{\left(e_{B}^{(1)}, r_{B}^{(1)}\right)}\left\{\delta \mathbb{E}\left[\pi_{B}^{(2) *}\left(\tilde{r}_{A}^{(2)}\right)\right]+\alpha\left(e_{B}^{(1)}\right)^{2}\right. \\
& +\left(r_{A}^{(1)}+\nu_{A} \sum_{n=2}^{4} \tilde{e}_{A}^{(n)}+\left(1-\nu_{A}\right)\left(e_{B}^{(1)}+\sum_{n=2}^{4} \tilde{e}_{B}^{(n)}-0.5\right)\right) \\
& \left.\cdot\left(r_{B}^{(1)}-r_{A}^{(1)}\right)+2\left(\left(r_{A}^{(1)}\right)^{2}-\left(r_{B}^{(1)}\right)^{2}\right)\right\}, \\
\mathbb{E}\left[\pi_{B}^{(2) *}\left(r_{A}^{(2)}\right)\right]= & \max _{\left(e_{B}^{(2)}, r_{B}^{(2)}\right)}\left\{\delta \mathbb{E}\left[\pi_{B}^{(3) *}\left(\tilde{r}_{A}^{(3)}\right)\right]+\alpha\left(e_{B}^{(2)}\right)^{2}\right. \\
& +\left(2 r_{A}^{(2)}+\nu_{A} \sum_{n=3}^{4} \tilde{e}_{A}^{(n)}+\left(1-\nu_{A}\right)\left(e_{B}^{(2)}+\sum_{n=3}^{4} \tilde{e}_{B}^{(n)}-0.5\right)\right) \\
& \left.\cdot\left(r_{B}^{(2)}-r_{A}^{(2)}\right)+2\left(\left(r_{A}^{(2)}\right)^{2}-\left(r_{B}^{(2)}\right)^{2}\right)\right\} \\
\mathbb{E}\left[\pi_{B}^{(3) *}\left(r_{A}^{(3)}\right)\right]= & \max _{\left(e_{B}^{(3)}, r_{B}^{(3)}\right)}\left\{\delta \mathbb{E}_{B}^{(4) *}\left(\tilde{r}_{A}^{(4)}\right)\right]+\alpha\left(e_{B}^{(3)}\right)^{2} \\
& +\left(3 r_{A}^{(3)}+\nu_{A} \tilde{e}_{A}^{(4)}+\left(1-\nu_{A}\right)\left(e_{B}^{(3)}+\tilde{e}_{B}^{(4)}-0.5\right)\right) \\
& \left.\cdot\left(r_{B}^{(3)}-r_{A}^{(3)}\right)+2\left(\left(r_{A}^{(3)}\right)^{2}-\left(r_{B}^{(3)}\right)^{2}\right)\right\} . \\
& \left.+2\left(\left(r_{A}^{(4)}\right)^{2}-\left(r_{B}^{(4)}\right)^{2}\right)\right\} . \\
\mathbb{E}\left[\pi_{B}^{(4) *}\left(r_{A}^{(4)}\right)\right]= & \max _{\left(e_{B}^{(4)}, r_{B}^{(4)}\right)}\left\{\alpha\left(e_{B}^{(4)}\right)^{2}\right. \\
+ & \left(4 r_{A}^{(4)}+\left(1-\nu_{A}\right)\left(e_{B}^{(4)}-0.5\right)\right)\left(r_{B}^{(4)}-r_{A}^{(4)}\right) \\
+ & 29 \mathrm{~d}) \\
&
\end{aligned}
$$

Lemma 10. If $\alpha>\max \left\{\frac{\left(1-\nu_{A}\right)^{2}}{2}, \frac{\nu_{A}^{2}}{2}\right\}$ and $r_{A}^{(4)} \leqslant \frac{7+\nu_{A}}{8}$, then $\left(e_{B}^{(4) *}, r_{B}^{(4) *}\right)=$ $\left(1, r_{A}^{(4)}+\frac{1}{8}\left(1-\nu_{A}\right)\right)$ and $\mathbb{E}\left[\pi_{B}^{(4) *}\left(r_{A}^{(4)}\right)\right]=\alpha+\frac{1}{8}\left(1-\nu_{A}\right)^{2}$.

Proof. When $i=4$, there are no future rounds. Consider Bob's function of expected payoff scores in round 4 as $f_{B}^{(4)}$ :

$$
\begin{aligned}
f_{B}^{(4)}=\mathbb{E}\left[\pi_{B}^{(4)}\left(r_{A}^{(4)}, a_{B}^{(4)}\right)\right] \\
=\alpha\left(e_{B}^{(4)}\right)^{2}+\left(4 r_{A}^{(4)}+\left(1-\nu_{A}\right)\left(e_{B}^{(4)}-0.5\right)\right)\left(r_{B}^{(4)}-r_{A}^{(4)}\right) \\
+2\left(\left(r_{A}^{(4)}\right)^{2}-\left(r_{B}^{(4)}\right)^{2}\right)
\end{aligned}
$$




\begin{tabular}{|c|c|c|}
\hline$e_{B}^{(4)}$ & $r_{B}^{(4)}$ & $f_{B}^{(4)}$ \\
\hline 0 & $r_{A}^{(4)}+\frac{1}{8}\left(\nu_{A}-1\right)$ & $\frac{1}{32}\left(1-\nu_{A}\right)^{2}$ \\
\hline 1 & $r_{A}^{(4)}+\frac{1}{8}\left(1-\nu_{A}\right)$ & $\alpha+\frac{1}{8}\left(1-\nu_{A}\right)^{2}$ \\
\hline$\frac{r_{A}^{(4)}\left(1-\nu_{A}\right)}{2 \alpha}$ & 0 & $-2\left(r_{A}^{(4)}\right)^{2}+\frac{r_{A}^{(4)}}{2}\left(1-\nu_{A}\right)+\frac{\left(1-\nu_{A}\right)^{2}}{4 \alpha}\left(1-\frac{r_{A}^{(4)}}{2}\right)$ \\
\hline 0 & 0 & $-2\left(r_{A}^{(4)}\right)^{2}+\frac{r_{A}^{(4)}}{2}\left(1-\nu_{A}\right)$ \\
\hline 0 & 1 & $-2\left(r_{A}^{(4)}\right)^{2}+4 r_{A}^{(4)}-2-\frac{\left(1-\nu_{A}\right)\left(1-r_{A}^{(4)}\right)}{2} \leqslant 0$ \\
\hline 1 & 0 & $-2\left(r_{A}^{(4)}\right)^{2}-\frac{\left(1-\nu_{A}\right) r_{A}^{(4)}}{2}+\alpha \leqslant \alpha$ \\
\hline 1 & 1 & $\alpha-2\left(1-r_{A}^{(4)}\right)^{2}+\frac{\left(1-\nu_{A}\right)\left(1-r_{A}^{(4)}\right)}{2}$ \\
\hline
\end{tabular}

Table 8: KKT points of $f_{B}^{(4)}$ in case 4

Then we obtain

$$
\nabla^{2} f_{B}^{(4)}\left(e_{B}^{(4)}, r_{B}^{(4)}\right)=\left[\begin{array}{ll}
\frac{\partial^{2} f_{B}^{(4)}}{\partial\left(e_{B}^{(4)}\right)^{2}} & \frac{\partial^{2} f_{B}^{(4)}}{\partial e_{B}^{(4)} \partial r_{B}^{(4)}} \\
\frac{\partial^{2} f_{B}^{(4)}}{\partial r_{B}^{(4)} \partial e_{B}^{(4)}} & \frac{\partial^{2} f_{B}^{(4)}}{\partial\left(r_{B}^{(4)}\right)^{2}}
\end{array}\right]=\left[\begin{array}{lr}
2 \alpha & 1-\nu_{A} \\
1-\nu_{A} & -4
\end{array}\right]
$$

The first principle minor of $\nabla^{2} f_{B}^{(4)}\left(e_{B}^{(4)}, r_{B}^{(4)}\right)$ is positive because $\operatorname{det}\left[\frac{\partial^{2} f_{B}^{(4)}}{\partial\left(e_{B}^{(4)}\right)^{2}}\right]=$ $2 \alpha>0$. the second principle minor is negative because:

$$
\operatorname{det}\left[\begin{array}{cc}
\frac{\partial^{2} f_{B}^{(4)}}{\partial\left(e_{B}^{(4)}\right)^{2}} & \frac{\partial^{2} f_{B}^{(4)}}{\partial e_{B}^{(4)} \partial r_{B}^{(4)}} \\
\frac{\partial^{2} f_{B}^{(4)}}{\partial r_{B}^{(4)} \partial e_{B}^{(4)}} & \frac{\partial^{2} f_{B}^{(4)}}{\partial\left(r_{B}^{(4)}\right)^{2}}
\end{array}\right]=-8 \alpha-\left(1-\nu_{A}\right)^{2}<0 .
$$

We find that the Hessian of $f_{B}^{(4)}$ is an indefinite matrix so we still need to find its KKT points and compare the corresponding values of $f_{B}^{(4)}$, for the maximal value $f_{B}^{(4) *}$ must be attained in one of these KKT points. We write down in table 8 the KKT points of $f_{B}^{(4)}$ and the corresponding function values. However, to simplify our analysis, unfeasible KKT points are not shown in this table. We find that if $r_{A}^{(4)} \leqslant \frac{7+\nu_{A}}{8}, f_{B}^{(4) *}$ is attained as $\alpha+$ $\frac{1}{8}\left(1-\nu_{A}\right)^{2}$ when $\left(e_{B}^{(4)}, r_{B}^{(4)}\right)=\left(1, r_{A}^{(4)}+\frac{1}{8}\left(1-\nu_{A}\right)\right)$. So Lemma 10 is true. 
Lemma 11. If $\alpha>\max \left\{\frac{\left(1-\nu_{A}\right)^{2}}{2}, \frac{\nu_{A}^{2}}{2}\right\}$ and $r_{A}^{(4)} \geqslant \frac{7+\nu_{A}}{8}$, then $\left(e_{B}^{(4) *}, r_{B}^{(4) *}\right)=$ $(1,1)$ and $\mathbb{E}\left[\pi_{B}^{(4) *}\left(r_{A}^{(4)}\right)\right]=\alpha-2\left(1-r_{A}^{(4)}\right)^{2}+\frac{\left(1-\nu_{A}\right)\left(1-r_{A}^{(4)}\right)}{2}$.

Proof. Similar as the proof for Lemma 10, if $r_{A}^{(4)} \geqslant \frac{7+\nu_{A}}{8}$, by comparing the values of $f_{B}^{(4)}$ of feasible KKT points in table 8 , we find that $f_{B}^{(4) *}$ is attained as $f_{B}^{(4) *}=\alpha-2\left(1-r_{A}^{(4)}\right)^{2}+\frac{\left(1-\nu_{A}\right)\left(1-r_{A}^{(4)}\right)}{2}$ when $\left(e_{B}^{(4) *}, r_{B}^{(4) *}\right)=(1,1)$. So Lemma 11 is true.

Given the two cases for Bob's equilibrium decisions in round 4, we may now determine Bob's round 4 decisions:

Theorem 9. If high efforts bring net rewards and $\alpha>\max \left\{\frac{\left(1-\nu_{A}\right)^{2}}{2}, \frac{\nu_{A}^{2}}{2}\right\}$, then

$$
\begin{gathered}
\left(e_{B}^{(4) *}, r_{B}^{(4) *}\right)= \begin{cases}\left(1, r_{A}^{(4)}+\frac{1}{8}\left(1-\nu_{A}\right)\right) & \text { if } 0 \leqslant r_{A}^{(4)} \leqslant \frac{7+\nu_{A}}{8} \\
(1,1) & \text { if } \frac{7+\nu_{A}}{8} \leqslant r_{A}^{(4)} \leqslant 1\end{cases} \\
f_{B}^{(4) *}= \begin{cases}\alpha+\frac{1}{8}\left(\nu_{A}-1\right)^{2} & \text { if } 0 \leqslant r_{A}^{(4)} \leqslant \frac{7+\nu_{A}}{8} \\
\alpha-2\left(1-r_{A}^{(4)}\right)^{2}+\frac{\left(1-\nu_{A}\right)\left(1-r_{A}^{(4)}\right)}{2} & \text { if } \frac{7+\nu_{A}}{8} \leqslant r_{A}^{(4)} \leqslant 1\end{cases}
\end{gathered}
$$

Proof. With Lemma 10 and Lemma 11, Theorem 9 is true.

We now move to Alice's optimal actions in round 4. By Theorem 9 we know that Bob would always exert high effort in round 4 . In other words Alice would assume $\tilde{e}_{B}^{(4)}=1$ when calculating her maximal expected payoff in round 4. We find that Alice will exert high effort and report as 1 in round 4 no matter what $B$ ob reports in round 3.

Theorem 10. If high efforts bring net rewards and $\alpha>\max \left\{\frac{\left(1-\nu_{A}\right)^{2}}{2}, \frac{\nu_{A}^{2}}{2}\right\}$, Alice's optimal action set in round 4 is $\left(e_{A}^{(4) *}, r_{A}^{(4) *}\right)=\left(1, \frac{3 r_{B}^{(3)}+1}{4}\right)$, and the optimal expected payoff score of round 4 is $\mathbb{E}\left[\pi_{A}^{(4) *}\left(r_{B}^{(3)}\right)\right]=\alpha+\frac{1}{8}\left(r_{B}^{(3)}-1\right)^{2}$.

Proof. Consider Alice's function of expected payoff scores in round 4 as $f_{A}^{(4)}$ :

$$
\begin{aligned}
f_{A}^{(4)}= & \mathbb{E}\left[\pi_{A}^{(4)}\left(r_{B}^{(3)}, a_{A}^{(4)}\right)\right] \\
= & \alpha\left(e_{A}^{(4)}\right)^{2}+\left(3 \cdot r_{B}^{(3)}+\nu_{A} e_{A}^{(4)}+\left(1-\nu_{A}\right) \tilde{e}_{B}^{(4)}\right)\left(r_{A}^{(4)}-r_{B}^{(3)}\right) \\
& +2\left(\left(r_{B}^{(3)}\right)^{2}-\left(r_{A}^{(4)}\right)^{2}\right) .
\end{aligned}
$$




\begin{tabular}{|c|c|c|}
\hline$e_{A}^{(4)}$ & $r_{A}^{(4)}$ & $f_{A}^{(4)}$ \\
\hline \hline 0 & $\frac{3 r_{B}^{(3)}+1-\nu_{A}}{4}$ & $\frac{1}{8}\left(r_{B}^{(3)}+\nu_{A}-1\right)^{2}$ \\
\hline 1 & $\frac{3 r_{B}^{(3)}+1}{4}$ & $\alpha+\frac{1}{8}\left(r_{B}^{(3)}-1\right)^{2}$ \\
\hline$\frac{\nu_{A} r_{B}^{(B)}}{2 \alpha}$ & 0 & $r_{B}^{(3)}\left(\nu_{A}-r_{B}^{(3)}-1\right)-\frac{\left(\nu_{A} r_{B}^{(3)}\right)^{2}}{4 \alpha}$ \\
\hline 0 & 0 & $r_{B}^{(3)}\left(\nu_{A}-1-r_{B}^{(3)}\right)$ \\
\hline 0 & 1 & $\left(1-r_{B}^{(3)}\right)\left(r_{B}^{(3)}-1-\nu_{A}\right)$ \\
\hline 1 & 0 & $\alpha-r_{B}^{(3)}\left(1+r_{B}^{(3)}\right)$ \\
\hline 1 & 1 & $\alpha-\left(1-r_{B}^{(3)}\right)^{2}$ \\
\hline
\end{tabular}

Table 9: KKT points of $f_{A}^{(4)}$ in case 4

After inserting $\tilde{e}_{B}^{(4)}=1$ into the above equation we can further get:

$$
\begin{aligned}
f_{A}^{(4)}= & \alpha\left(e_{A}^{(4)}\right)^{2}+\left(3 \cdot r_{B}^{(3)}+\nu_{A} \cdot e_{A}^{(4)}+\left(1-\nu_{A}\right)\right)\left(r_{A}^{(4)}-r_{B}^{(3)}\right) \\
& +2\left(\left(r_{B}^{(3)}\right)^{2}-\left(r_{A}^{(4)}\right)^{2}\right) .
\end{aligned}
$$

To find the value of $f_{A}^{(4) *}$, we need to check the Hessian of function $f_{A}^{(4)}$ :

$$
\nabla^{2} f_{A}^{(4)}\left(e_{A}^{(4)}, r_{A}^{(4)}\right)=\left[\begin{array}{cc}
\frac{\partial^{2} f_{A}^{(4)}}{\partial\left(e_{A}^{(4)}\right)^{2}} & \frac{\partial^{2} f_{A}^{(4)}}{\partial e_{A}^{(4)} \partial r_{A}^{(4)}} \\
\frac{\partial^{2} f_{A}^{(4)}}{\partial r_{A}^{(4)} \partial e_{A}^{(4)}} & \frac{\partial^{2} f_{A}^{(4)}}{\partial\left(r_{A}^{(4)}\right)^{2}}
\end{array}\right]=\left[\begin{array}{cc}
2 \alpha & \nu_{A} \\
\nu_{A} & -4
\end{array}\right]
$$

We find that the Hessian of $f_{A}^{(4)}$ is also an indefinite matrix because the value of its first principle minor is positive as $2 \alpha>0$, and the value of its second principle minor is negative as $-8 \alpha-\nu_{A}^{2}<0$. We write down in table 9 the KKT points of $f_{A}^{(4)}$ and the corresponding function values. We find that $f_{A}^{(4)}$ is maximized as $f_{A}^{(4) *}=\alpha+\frac{1}{8}\left(r_{B}^{(3)}-1\right)^{2}$ when $\left(e_{A}^{(4)}, r_{A}^{(4)}\right)=\left(1, \frac{3 r_{B}^{(3)}+1}{4}\right)$. So Theorem 10 is true.

Consider Bob in round 3. From Theorem 9 and Theorem 10 we have $e_{A}^{(4) *}=e_{B}^{(4) *}=1$. Knowing $\tilde{e}_{A}^{(4)}=\tilde{e}_{B}^{(4)}=1$, we get the expression of Bob's 
expected payoff score in round 3 as $f_{B}^{(3)}$ :

$$
\begin{aligned}
f_{B}^{(3)}=\alpha & \cdot\left(e_{B}^{(3)}\right)^{2} \\
+ & \left(3 r_{A}^{(3)}-0.5\left(1-\nu_{A}\right)+\nu_{A}+\left(1-\nu_{A}\right) \cdot\left(e_{B}^{(3)}+1\right)\right) \\
& \cdot\left(r_{B}^{(3)}-r_{A}^{(3)}\right)+2\left(\left(r_{A}^{(3)}\right)^{2}-\left(r_{B}^{(3)}\right)^{2}\right)
\end{aligned}
$$

Lemma 12. If high efforts bring net rewards and $\alpha>\max \left\{\frac{\left(1-\nu_{A}\right)^{2}}{2}, \frac{\nu_{A}^{2}}{2}\right\}$, then

$$
\begin{gathered}
\left(e_{B}^{(4) *}, r_{B}^{(4) *}\right)= \begin{cases}\left(1, \frac{3 r_{B}^{(3)}+1}{4}+\frac{1}{8}\left(1-\nu_{A}\right)\right), & \text { if } 0 \leqslant r_{B}^{(3)} \leqslant \frac{5+\nu_{A}}{6} \\
(1,1), & \text { if } \frac{5+\nu_{A}}{6} \leqslant r_{B}^{(3)} \leqslant 1\end{cases} \\
f_{B}^{(4) *}= \begin{cases}\alpha+\frac{1}{8}\left(1-\nu_{A}\right)^{2} & \text { if } 0 \leqslant r_{B}^{(3)} \leqslant \frac{5+\nu_{A}}{6} \\
\alpha+\frac{3\left(1-r_{B}^{(3)}\right)\left(3 r_{B}^{(3)}-\nu_{A}-2\right)}{8} & \text { if } \frac{5+\nu_{A}}{6} \leqslant r_{B}^{(3)} \leqslant 1\end{cases}
\end{gathered}
$$

Proof. According to Theorem 10, we know that $r_{A}^{(4) *}=\frac{3 r_{B}^{(3)}+1}{4}$. Together with the statement of Theorem 9, we infer Lemma 12.

Lemma 13. If $\alpha>\max \left\{\frac{\left(1-\nu_{A}\right)^{2}}{2}, \frac{\nu_{A}^{2}}{2}\right\}$ and $r_{A}^{(3)} \leqslant \frac{7 \nu_{A}+11}{18}$, then $\left(e_{B}^{(3) *}, r_{B}^{(3) *}\right)=$ $\left(1, \frac{3}{4} r_{A}^{(3)}+\frac{3-\nu_{A}}{8}\right)$ and

$$
\begin{aligned}
\mathbb{E}\left[\pi_{B}^{(3) *}\left(r_{A}^{(3)}\right)\right]= & \alpha+\frac{r_{A}^{(3)}}{8}\left(r_{A}^{(3)}+\nu_{A}-3\right)+\frac{\left(\nu_{A}-3\right)^{2}}{32} \\
& +\frac{9}{8}+\delta\left(\alpha+\frac{1}{8}\left(\nu_{A}-1\right)^{2}\right) .
\end{aligned}
$$

Proof. From Lemma 12 we know that $f_{B}^{(4) *}$ is attained as $f_{B}^{(4) *}=\alpha+\frac{1}{8}(1-$ $\left.\nu_{A}\right)^{2}$ when $r_{B}^{(3)} \leqslant \frac{5+\nu_{A}}{6}$; and attained as $f_{B}^{(4) *}=\alpha+\frac{3\left(1-r_{B}^{(3)}\right)\left(3 r_{B}^{(3)}-\nu_{A}-2\right)}{8}$ when $r_{B}^{(3)} \geqslant \frac{5+\nu_{A}}{6}$.

We first discuss the situation where $f_{B}^{(4) *}$ is a constant as $f_{B}^{(4) *}=\alpha+$ $\frac{1}{8}\left(\nu_{A}-1\right)^{2}$ when $r_{B}^{(3)} \leqslant \frac{5+\nu_{A}}{6}$. Similarly we denote the KKT points of $f_{B}^{(3)}+\delta \cdot f_{B}^{(4) *}$ in table 10. We find that when $r_{A}^{(3)} \leqslant \frac{7 \nu_{A}+11}{18}, f_{B}^{(3)}+\delta \cdot f_{B}^{(4) *}$ is maximized as $\alpha+\frac{r_{A}^{(3)}}{8}\left(r_{A}^{(3)}+\nu_{A}-3\right)+\frac{\left(\nu_{A}-3\right)^{2}}{32}+\frac{9}{8}+\delta\left(\alpha+\frac{1}{8}\left(\nu_{A}-1\right)^{2}\right)$, which we denote as $\mathbb{E}\left[\pi_{B}^{(3)}\left(r_{A}^{(3)}, a_{B}^{(3)}\right)\right]$, when $\left(e_{B}^{(3)}, r_{B}^{(3)}\right)=\left(1, \frac{3}{4} r_{A}^{(3)}+\frac{3-\nu_{A}}{8}\right)$.

Second, we discuss the situation where $f_{B}^{(4) *}$ is a function of $r_{B}^{(3)}$ as $f_{B}^{(4) *}=$ $\alpha+\frac{3\left(1-r_{B}^{(3)}\right)\left(3 r_{B}^{(3)}-\nu_{A}-2\right)}{8}$, if $r_{B}^{(3)} \geqslant \frac{5+\nu_{A}}{6}$. Using the same method, we find that 


\begin{tabular}{|c|c|c|}
\hline$e_{B}^{(3)}$ & $r_{B}^{(3)}$ & $f_{B}^{(3)}+\delta \cdot f_{B}^{(4) *}$ \\
\hline \hline 0 & $\frac{3}{4} r_{A}^{(3)}+\frac{1}{8}\left(\nu_{A}+1\right)$ & $\begin{array}{c}\frac{1}{8} r_{A}^{(3)}\left(r_{A}^{(3)}-1-\nu_{A}\right)+\frac{1}{32}\left(\nu_{A}+1\right)^{2} \\
+\delta\left(\alpha+\frac{1}{8}\left(\nu_{A}-1\right)^{2}\right)\end{array}$ \\
\hline 1 & $\frac{3}{4} r_{A}^{(3)}+\frac{3-\nu_{A}}{8}$ & $\begin{array}{c}\alpha+\frac{r_{A}^{(3)}}{8}\left(r_{A}^{(3)}+\nu_{A}-3\right)+\frac{\left(\nu_{A}-3\right)^{2}}{32} \\
+\frac{9}{8}+\delta\left(\alpha+\frac{1}{8}\left(\nu_{A}-1\right)^{2}\right)\end{array}$ \\
\hline$\frac{r_{A}^{(3)}\left(1-\nu_{A}\right)}{2 \alpha}$ & 0 & $-\left(r_{A}^{(3)}\right)^{2}-\frac{r_{A}^{(3)}\left(\nu_{A}+1\right)}{2}-\frac{\left(r_{A}^{(3)}\right)^{2}\left(1-\nu_{A}\right)^{2}}{4 \alpha}$ \\
\hline 0 & 0 & $-r_{A}^{(3)}\left(r_{A}^{(3)}+\frac{\nu_{A}+1}{2}\right)+\delta\left(\alpha+\frac{1}{8}\left(\nu_{A}-1\right)^{2}\right)$ \\
\hline 1 & 0 & $\alpha-r_{A}^{(3)}\left(r_{A}^{(3)}+\frac{3-\nu_{A}}{2}\right)+\delta\left(\alpha+\frac{1}{8}\left(\nu_{A}-1\right)^{2}\right)$ \\
\hline
\end{tabular}

Table 10: KKT points of $f_{B}^{(3)}+\delta \cdot f_{B}^{(4) *}$ in case 4 when $r_{B}^{(3)} \leqslant \frac{5+\nu_{A}}{6}$

$f_{B}^{(3)}+\delta \cdot f_{B}^{(4) *}$ is maximized as $\alpha+\frac{r_{A}^{(3)}}{2}\left(3-2 r_{A}^{(3)}\right)+\frac{\nu_{A}\left(r_{A}^{(3)}-1\right)-1}{2}+\alpha(1+\delta)$ at $\left(e_{B}^{(3)}, r_{B}^{(3)}\right)=(1,1)$.

By routine calculations, we know that the first expression of $f_{B}^{(3)}+\delta$. $f_{B}^{(4) *}$ is larger than the value of the second expression. So $\mathbb{E}\left[\pi_{B}^{(3) *}\left(r_{A}^{(3)}\right)\right]$ is achieved as $\alpha+\frac{r_{A}^{(3)}}{8}\left(r_{A}^{(3)}+\nu_{A}-3\right)+\frac{\left(\nu_{A}-3\right)^{2}}{32}+\frac{9}{8}+\delta\left(\alpha+\frac{1}{8}\left(\nu_{A}-1\right)^{2}\right)$ at $\left(e_{B}^{(3) *}, r_{B}^{(3) *}\right)=\left(1, \frac{3}{4} r_{A}^{(3)}+\frac{3-\nu_{A}}{8}\right)$. So Lemma 13 is true.

Lemma 14. If $\alpha>\max \left\{\frac{\left(1-\nu_{A}\right)^{2}}{2}, \frac{\nu_{A}^{2}}{2}\right\}$ and $r_{A}^{(3)} \geqslant \frac{7 \nu_{A}+11}{18}$, then $\left(e_{B}^{(3) *}, r_{B}^{(3) *}\right)=$ $(1,1)$ and

$$
\mathbb{E}\left[\pi_{B}^{(3) *}\left(r_{A}^{(3)}\right)\right]=\alpha+\frac{r_{A}^{(3)}}{2}\left(3-2 r_{A}^{(3)}\right)+\frac{\nu_{A}\left(r_{A}^{(3)}-1\right)-1}{2}+\alpha(1+\delta) .
$$

Proof. The proof is similar to that of Lemma 13. Details are omitted.

Given the two cases for Bob's equilibrium decisions in round 3, we may now determine Bob's round 3 decisions:

Theorem 11. If high efforts bring net rewards and $\alpha>\max \left\{\frac{\left(1-\nu_{A}\right)^{2}}{2}, \frac{\nu_{A}^{2}}{2}\right\}$, then

$$
\left(e_{B}^{(3) *}, r_{B}^{(3) *}\right)= \begin{cases}\left(1, \frac{3}{4} r_{A}^{(3)}+\frac{3-\nu_{A}}{8}\right), & \text { if } 0 \leqslant r_{A}^{(3)} \leqslant \frac{7 \nu_{A}+11}{18} \\ (1,1), & \text { if } \frac{7 \nu_{A}+11}{18} \leqslant r_{A}^{(3)} \leqslant 1\end{cases}
$$




$$
f_{B}^{(3) *}=\left\{\begin{array}{rlrl}
\alpha & +\frac{r_{A}^{(3)}}{8}\left(r_{A}^{(3)}+\nu_{A}-3\right)+\frac{\left(\nu_{A}-3\right)^{2}}{32} & \\
& +\frac{9}{8}+\delta\left(\alpha+\frac{1}{8}\left(\nu_{A}-1\right)^{2}\right), & & \\
\alpha+\frac{r_{A}^{(3)}}{2}\left(3-2 r_{A}^{(3)}\right)+\frac{\nu_{A}\left(r_{A}^{(3)}-1\right)-1}{2}+\alpha(1+\delta), & & \text { if } \frac{7 \nu_{A}+11}{18} \leqslant r_{A}^{(3)} \leqslant 1
\end{array}\right.
$$

Proof. With Lemma 13 and Lemma 14, Theorem 11 is true.

Consider Alice in round 3. From Theorem 9, Theorem 10 and Theorem 11 we have $e_{B}^{(3) *}=e_{A}^{(4) *}=e_{B}^{(4) *}=1$. Knowing $\tilde{e}_{B}^{(3)}=\tilde{e}_{A}^{(4)}=\tilde{e}_{B}^{(4)}=1$, we get the expression of Alice's expected payoff score in round 3 as $f_{A}^{(3)}$ :

$$
\begin{aligned}
& f_{A}^{(3)}=\alpha\left(e_{A}^{(3)}\right)^{2}+\left(2 r_{B}^{(2)}+\nu_{A}\left(e_{A}^{(3)}+1\right)+2\left(1-\nu_{A}\right)\right)\left(r_{A}^{(3)}-r_{B}^{(2)}\right)-2\left(r_{A}^{(3)}\right)^{2}+2\left(r_{B}^{(2)}\right)^{2} \\
&=\alpha\left(e_{A}^{(3)}\right)^{2}+\left(2 r_{B}^{(2)}+\nu_{A} e_{A}^{(3)}+2-\nu_{A}\right)\left(r_{A}^{(3)}-r_{B}^{(2)}\right) \\
& \quad-2\left(r_{A}^{(3)}\right)^{2}+2\left(r_{B}^{(2)}\right)^{2}
\end{aligned}
$$

Theorem 12. If high efforts bring net rewards and $\alpha>\max \left\{\frac{\left(1-\nu_{A}\right)^{2}}{2}, \frac{\nu_{A}^{2}}{2}\right\}$, then

$$
\begin{gathered}
\left(e_{A}^{(3) *}, r_{A}^{(3) *}\right)= \begin{cases}\left(1, \frac{9 r_{B}^{(3)}+3}{16}+\frac{3-\nu_{A}}{8}\right), & \text { if } 0 \leqslant r_{A}^{(3)} \leqslant \frac{7 \nu_{A}+11}{18} \\
(1,1), & \text { if } \frac{7 \nu_{A}+11}{18} \leqslant r_{A}^{(3)} \leqslant 1\end{cases} \\
f_{A}^{(3) *}= \begin{cases}\alpha+\frac{1}{8}\left(\frac{3}{4} r_{A}^{(3)}+\frac{\nu_{A}-5}{8}\right)^{2} & \text { if } 0 \leqslant r_{A}^{(3)} \leqslant \frac{7 \nu_{A}+11}{18} \\
\alpha & \text { if } \frac{7 \nu_{A}+11}{18} \leqslant r_{A}^{(3)} \leqslant 1\end{cases}
\end{gathered}
$$

Proof. According to Theorem 11, we know that:

$$
r_{B}^{(3) *}= \begin{cases}\frac{3}{4} r_{A}^{(3)}+\frac{3-\nu_{A}}{8}, & \text { if } 0 \leqslant r_{A}^{(3)} \leqslant \frac{7 \nu_{A}+11}{18} \\ 1, & \text { if } \frac{7 \nu_{A}+11}{18} \leqslant r_{A}^{(3)} \leqslant 1\end{cases}
$$

Together with the statement of Theorem 10, we infer Lemma 12.

Algebraically finishing the proof is quite involved and lengthy, and will not bring any further insights. As such, we will finish the four round case using a combinatorial argument. So far we know that regardless of an agent's report, her action in round 3 and 4 both $A$ lice and $B$ ob will exert high efforts. We now consider Bob's actions in round 2. Being rational he deduces that all subsequent effort levels of all other agents, including himself will be to 
exert high effort. Thus, from his perspective at the end of round 2 he has no uncertainty regarding future effort levels, and all past effort levels are already fixed. As such, his only trade off is to either exert low effort, lose the reward from high effort, but make it up within in the prediction market. If $\alpha$ if sufficiently high, then the reward within the prediction market will be outweighed by the reward from exerting high effort. Assuming $\alpha$ is sufficiently high, then Bob will exert high effort and report truthfully.

Rolling forward to Alice's actions in round 2, we may apply the same combinatorial argument to Alice. Similarly, we may again apply the same arguments to $B$ ob and then Alice, in round 1. We in turn deduce that for $\alpha$ sufficiently high, all agents will exert high effort in all rounds and report their beliefs truthfully.

\section{Discussion and Conclusion}

In this work we show that the cost actions agents take that determine the outcome of a prediction market traded event has great influence on agents' prediction market and external behavior. We find that in a 2-round setting and later the 4-round setting, when agents are forward-looking and want to maximize their total expected payoffs from exerting efforts towards realizing the traded event as well as from trading in the prediction market, asymmetric action costs results in agents avoiding taking the costliest action. This observation implies: if a market maker rewards her preferred action more than a less preferred action, then agents will take the desired action even when a prediction market is present. We find that the value of the net reward to each desirable action should be larger than a certain amount, which is determined by the value of $\nu_{A}$ in our 2-round setting, ${ }^{2}$ in a sense the reward of desirable actions have to be sufficiently large, this makes sense as the "playing for peanuts results" are avoided (Harinck et al., 2007; Weber and Chapman, 2005). Perhaps unexpectedly, even though agents' actions are influenced by external costs, agents will always report truthfully during each prediction market round, given sufficiently high external rewards. This observation, shows that the quadratic market scoring rule is incentivecompatible even with external incentives present, so long as action costs are costly and the preferred action is adequately rewarded.

In the past, decision and policy makers have expressed concern that the existence of a prediction market will inspire undesirable actions for agents

\footnotetext{
${ }^{2}$ We did not explicitly determine the exact threshold for $\alpha$ in the 4-round setting. Just as in the 2-round setting this threshold exists and is a function of $\nu_{A}$.
} 
trading in the prediction market. Especially when the same agents trading have a direct impact on deciding the likelihood of the predicted event, as is the case in corporate settings. However, previous research does not take into account the potential payoffs (could be net costs or net rewards) to the agents who take such actions. We base our research on the assumption that forward-looking agents wish to maximize their total expected payoffs not only from the prediction market but also from their actions related to the traded event. With our finding, a market maker who cares about the result of the traded event can incentivize agents' to take desirable actions. More importantly, our results address the concern firms have regarding deploying a prediction market within their organization as the existence of such markets will not induce participants to change their behavior outside of the market, assuming they are appropriately compensated. A market maker can also gain accurate and true information about agents' actions from the same agents' reports in the prediction market, as the regular market scoring rule is incentive-compatible.

In this work we set a range for expected payoff scores of exerted efforts, whose absolute value should be larger than a certain value in each round, in order to incentivize desired actions and truthful reports. We do not discuss whether the prediction market will still be incentive-compatible or not when this range is violated. However, we do show that when efforts are not costly actions (payoff scores are 0 for exerted efforts), agents will bluff in the prediction market, a well known existing result. More importantly, in this work we set the number of rounds $(T)$ to be 2 or 4 , which guarantees our assumption that agents are forward-looking. However, we need to extend our model to a finite round setting with $T$ being a large number to see whether our conclusion still holds. In addition, the result we presented only considered the quadratic market scoring rule. By the nature of our result, the logarithmic market scoring rule cannot be used to verify our result. However, it is not clear if there are other scoring rules that may be used, especially: what members of the large class of scoring rules introduced by Gneiting and Raftery (2007) restore incentive compatibility when external incentives are present? The results of our paper should not be applied blindly, but we hope this study motivates laboratory and field experiments to determine if incentive compatibility is restored when the cost of external actions is considered. We view the results presented here as a first step in incorporating costs of external actions in prediction markets that are used within corporations today. 


\section{References}

\section{References}

Kenneth J. Arrow, Robert Forsythe, Michael Gorham, Robert Hahn, Robin Hanson, John O. Ledyard, Saul Levmore, Robert Litan, Paul Milgrom, Forrest D. Nelson, George R. Neumann, Marco Ottaviani, Thomas C. Schelling, Robert J. Shiller, Vernon L. Smith, Erik Snowberg, Cass R. Sunstein, Paul C. Tetlock, Philip E. Tetlock, Hal R. Varian, Justin Wolfers, and Eric Zitzewitz. The Promise of Prediction Markets. Science, 320(5878), 2008. URL http://science.sciencemag.org/content/320/ $5878 / 877$.

J. Eric Bickel. Some Comparisons among Quadratic, Spherical, and Logarithmic Scoring Rules. Decision Analysis, 4(2):49-65, jun 2007. ISSN 1545-8490. doi: 10.1287/deca.1070.0089. URL http://pubsonline. informs.org/doi/abs/10.1287/deca.1070.0089.

Glenn W. Brier. Verification of Forecasts Expressed in Terms of Probability. Monthly Weather Review, 78(1):1-3, jan 1950. ISSN 0027-0644. doi: 10.1175/1520-0493(1950)078<0001:VOFEIT $\rangle 2.0$.CO;2.

Fredrik Carlsson, Dinky Daruvala, and Olof Johansson-Stenman. Are People Inequality-Averse, or Just Risk-Averse? Economica, 72(287):375-396, aug 2005. doi: 10.1111/j.0013-0427.2005.00421.x. URL http://doi.wiley. $\mathrm{com} / 10.1111 / \mathrm{j} .0013-0427.2005 .00421 . \mathrm{x}$.

Mithun Chakraborty and Sanmay Das. On Manipulation in Prediction Markets When Participants Influence Outcomes Directly. Technical report, arXiv, jul 2014. URL http://arxiv.org/abs/1407.7015.

Mithun Chakraborty and Sanmay Das. Trading on a rigged game: Outcome manipulation in prediction markets. IJCAI International Joint Conference on Artificial Intelligence, 2016-Janua:158-164, 2016. ISSN 10450823.

Mithun Chakraborty, Sanmay Das, Allen Lavoie, Malik Magdon-Ismail, and Yonatan Naamad. Instructor rating markets. In Proceedings of the Twenty-Seventh AAAI Conference on Artificial Intelligence, pages 159-165, Bellevue, WA, 2013. AAAI Press. URL https://dl.acm.org/ citation. $c f m ?$ id=2891483.

Yiling Chen, Stanko Dimitrov, Rahul Sami, Daniel M. Reeves, David M. Pennock, Robin D. Hanson, Lance Fortnow, and Rica Gonen. Gaming Prediction Markets: Equilibrium Strategies with a Market Maker. 
Algorithmica, 58(4):930-969, dec 2010. ISSN 0178-4617. doi: 10. 1007/s00453-009-9323-2. URL http://link.springer.com/10.1007/ s00453-009-9323-2.

Yiling Chen, Xi A Gao, Rick Goldstein, and Ian A Kash. Market manipulation with outside incentives. Autonomous Agents and Multi-Agent Systems, pages 1-36, 2014. doi: 10.1007/s10458-014-9249-1. URL http://dx.doi.org/10.1007/s10458-014-9249-1.

Sanmay Das. The effects of market-making on price dynamics. In Proceedings of the 7th International Joint Conference on Autonomous Agents and Multiagent Systems - Volume 2, AAMAS '08, pages 887-894, Richland, SC, 2008. International Foundation for Autonomous Agents and Multiagent Systems. ISBN 978-0-9817381-1-6. URL http://dl.acm.org/ citation. cfm?id=1402298.1402347.

Stanko Dimitrov and Rahul Sami. Non-myopic strategies in prediction markets. In Proceedings of the 9th ACM conference on Electronic commerce - EC '08, page 200, New York, New York, USA, 2008. ACM Press. ISBN 9781605581699. doi: 10.1145/1386790.1386823. URL http://portal.acm.org/citation. cfm?doid=1386790.1386823.

Stanko Dimitrov and Rahul Sami. Composition of Markets with Conflicting Incentives. In The Eleventh ACM Conference on Electronic Commerce (EC'10), jun 2010.

Stanko Dimitrov, Rahul Sami, and Marina A. Epelman. Subsidized Prediction Mechanisms for Risk Averse Agents. ACM Transactions on Economics and Computation, 3(4):1-23, jul 2015. doi: 10.1145/2716327. URL http: //dl.acm.org/citation. cfm?doid=2810066.2716327.

Tilmann Gneiting and Adrian E Raftery. Strictly Proper Scoring Rules, Prediction, and Estimation. Journal of the American Statistical Association, 102(477):359-378, mar 2007. ISSN 0162-1459. doi: 10.1198/ 016214506000001437. URL http://www . tandfonline.com/doi/abs/10. 1198/016214506000001437.

Andreas Graefe, J. Scott Armstrong, Randall J. Jones, and Alfred G. Cuzán. Combining forecasts: An application to elections. International Journal of Forecasting, 30(1):43-54, 2014. ISSN 01692070. doi: 10.1016/j.ijforecast. 2013.02.005. URL http://dx.doi.org/10.1016/j.ijforecast.2013. 02.005 . 
A. M. Hanea, M. F. McBride, M. A. Burgman, B. C. Wintle, F. Fidler, L. Flander, C. R. Twardy, B. Manning, and S. Mascaro. I nvestigate D iscuss E stimate A ggregate for structured expert judgement. International Journal of Forecasting, 33(1):267-279, 2017. ISSN 01692070. doi: 10. 1016/j.ijforecast.2016.02.008.

Robin Hanson. Logarithmic Market Scoring Rules for Modular Combinatorial Information Aggregation. Technical report, George Mason Univesity, Fairfax, VA, 2002.

Fieke Harinck, Eric Van Dijk, Ilja Van Beest, and Paul Mersmann. When Gains Loom Larger Than Losses. Psychological Science, 18(12):10991105, dec 2007. doi: 10.1111/j.1467-9280.2007.02031.x. URL http:// journals.sagepub.com/doi/10.1111/j.1467-9280.2007.02031.x.

Charles A Holt and Susan K Laury. Risk Aversion and Incentive Effects. American Economic Review, 92(5):1644-1655, nov 2002. ISSN 0002-8282. doi: 10.1257/000282802762024700. URL http://pubs . aeaweb.org/doi/ $10.1257 / 000282802762024700$.

EH Huang and Y Shoham. Price manipulation in prediction markets: analysis and mitigation. In $A A M A S$ '14: proceedings of the 2014 International Conference on Autonomous Agents $\&$ Multiagent Systems, number Aamas, pages 213-220, 2014. ISBN 978-1-4503-2738-1. URL http://dl.acm.org/citation. cfm?id=2615768.

Majid Karimi and Stanko Dimitrov. On the Road to Making Science of "Art": Risk Bias in Market Scoring Rules. Decision Analysis, 15(2), 2018 .

Robert E. Looney. DARPA's Policy Analysis Market for Intelligence: Outside the Box or Off the Wall? International Journal of Intelligence and CounterIntelligence, 17(3):405-419, sep 2004. ISSN 0885-0607. doi: 10.1080/08850600490446745. URL http://www.tandfonline.com/doi/ abs/10.1080/08850600490446745.

Edgar C. Merkle and Mark Steyvers. Choosing a Strictly Proper Scoring Rule. Decision Analysis, 10(4):292-304, dec 2013. ISSN 1545-8490. doi: 10.1287/deca.2013.0280. URL http://pubsonline.informs.org/doi/ abs/10.1287/deca.2013.0280. 
Marco Ottaviani and Peter Norman Sørensen. Outcome Manipulation in Corporate Prediction Markets. Journal of the European Economic Association, 5(2-3):554-563, may 2007. ISSN 1542-4766. doi: 10.1162/ jeea.2007.5.2-3.554. URL https://academic.oup.com/jeea/article/ 2295776/Outcome.

Thomas Peeters. Testing the Wisdom of Crowds in the field: Transfermarkt valuations and international soccer results. International Journal of Forecasting, 34(1):17-29, January 2018. ISSN 01692070. doi: 10.1016/j.ijforecast.2017.08.002. URL http://dx.doi.org/10.1016/j. ijforecast.2017.08.002.

PredictIt. How it Works. https://www.predictit.org/About/ HowItWorks, 2014. Accessed: 2018-04-30.

Alexandra G. Rosati and Brian Hare. Reward currency modulates human risk preferences. Evolution and Human Behavior, 37(2):159-168, mar 2016. ISSN 10905138. doi: 10.1016/j.evolhumbehav.2015.10.003. URL http://dx.doi.org/10.1016/j.evolhumbehav.2015.10.003http: //linkinghub.elsevier.com/retrieve/pii/S1090513815001063.

David Rothschild. Combining forecasts for elections: Accurate, relevant, and timely. International Journal of Forecasting, 31(3):952-964, 2015. ISSN 01692070. doi: 10.1016/j.ijforecast.2014.08.006. URL http://dx. doi.org/10.1016/j.ijforecast.2014.08.006.

Peng Shi, Vincent Conitzer, and Mingyu Guo. Prediction Mechanisms That Do Not Incentivize Undesirable Actions. In WINE'09, the fifth Workshop on Internet and Network Economics, pages 89-100, dec 2009. ISBN 978-3642-10840-2. doi: 10.1007/978-3-642-10841-9_10. URL http://dx.doi. org/10.1007/978-3-642-10841-9\{_\}10.

Bethany J. Weber and Gretchen B. Chapman. Playing for peanuts: Why is risk seeking more common for low-stakes gambles? Organizational Behavior and Human Decision Processes, 97(1):31-46, may 2005. doi: 10. 1016/J.OBHDP.2005.03.001. URL https://www.sciencedirect.com/ science/article/pii/S0749597805000361.

\section{Appendix A. Notation}

$\alpha$ constant that represents amount of cost (reward) for efforts, we assume $0<\alpha \leqslant 2$. 
$i$ number of round, $i=1, \ldots, T$.

$r_{A}^{(i)}$ the reported prediction probability of E's occurring in round $i$ if the report has already been made by $A$ lice, $r_{A}^{(i)} \in[0,1], \bar{r}_{A}^{(i)}=1-r_{A}^{(i)}$.

$r_{B}^{(i)}$ the reported prediction probability of $E$ 's occurring in round $i$ if the report has already been made by $B$ ob, $r_{B}^{(i)} \in[0,1], \bar{r}_{B}^{(i)}=1-r_{B}^{(i)}$.

$\tilde{r}_{A}^{(i)}$ the reported prediction probability of $E$ 's occurring in round $i$ if the report has not yet been made by Alice.

$\tilde{r}_{B}^{(i)}$ the reported prediction probability of $E$ 's occurring in round $i$ if the report has not yet been made by $B$ ob.

$r_{B}^{(0)}$ the beginning market estimate set in the prediction market by the market maker.

$e_{A}^{(i)}$ value of Alice's effort in round $i$ if the effort action has already happened, $e_{A}^{(1)}=1$ if Alice devotes high effort in round $i$, otherwise 0 , $\bar{e}_{A}^{(i)}=1-e_{A}^{(i)}$.

$e_{B}^{(i)}$ value of Bob's effort in round $i$ if the effort action has already happened, $e_{B}^{(1)}=1$ if Bob devotes high effort in round $i$, otherwise 0, $\bar{e}_{B}^{(i)}=1-e_{B}^{(i)}$.

$\tilde{e}_{A}^{(i)}$ value of Alice's future effort in round $i$ if the effort action has not yet taken place.

$\tilde{e}_{B}^{(i)}$ value of Bob's future effort in round $i$ if the effort action has not yet taken place.

$\mathbb{E}_{A}\left(\tilde{e}_{B}^{(i)}\right)$ the expectation of Bob's future effort value of round $i$ perceived by $A$ lice when she reports in round $i$.

$a_{A}^{(i)}$ the action set that $A$ lice takes in round $i, a_{A}^{(i)}=\left(e_{A}^{(i)}, r_{A}^{(i)}\right)$.

$a_{A}^{(i) *}$ the optimal action set that $A$ lice takes in round $i, a_{A}^{(i)}=\left(e_{A}^{(i) *}, r_{A}^{(i) *}\right)$.

$a_{A}$ the policy that $A$ lice takes for all rounds, $a_{A}:=\left(a_{A}^{(1)}, \ldots, a_{A}^{(T)}\right)$.

$a_{A}^{*}$ the optimal policy for Alice, $a_{A}^{*}:=\left(a_{A}^{(1) *}, \ldots, a_{A}^{(T) *}\right)$. 
$\mathcal{A}_{A}^{(i)}$ the action set that $A$ lice has in round $i, a_{A}^{(i)} \in \mathcal{A}_{A}^{(i)}$.

$\mathcal{A}_{A}$ the whole action set that $A$ lice has, $\mathcal{A}_{A}=\mathcal{A}_{A}^{(1)} \times \ldots \times \mathcal{A}_{A}^{(i)}$ and $a_{A} \in \mathcal{A}_{A}$.

$a_{B}^{(i)}$ the action set that $B$ ob takes in round $i, a_{B}^{(i)}=\left(e_{B}^{(i)}, r_{B}^{(i)}\right)$.

$a_{B}^{(i) *}$ the optimal action set that $B$ ob takes in round $i, a_{B}^{(i) *}=\left(e_{B}^{(i) *}, r_{B}^{(i) *}\right)$.

$a_{B}$ the policy that $B$ ob takes for all rounds, $a_{B}:=\left(a_{B}^{(1)}, \ldots, a_{B}^{(T)}\right)$.

$a_{B}^{*}$ the optimal policy for $B$ ob, $a_{B}^{*}:=\left(a_{B}^{(1) *}, \ldots, a_{B}^{(T) *}\right)$.

$\mathcal{A}_{B}^{(i)}$ the action set that $B$ ob has in round $i, a_{B}^{(i)} \in \mathcal{A}_{B}^{(i)}$.

$\mathcal{A}_{B}$ the whole action set that Bob has, $\mathcal{A}_{B}=\mathcal{A}_{B}^{(1)} \times \ldots \times \mathcal{A}_{B}^{(i)}$ and $a_{B} \in \mathcal{A}_{B}$.

$h_{A}^{(i)}$ number of high efforts Alice has exerted in total from round 1 to round $i, h_{A}^{(0)}=0$.

$h_{B}^{(i)}$ number of high efforts Bob has exerted in total from round 1 to round $i, h_{B}^{(0)}=0$.

$\nu_{A}$ Alice's impact on deciding the likelihood of project $E$ 's occurring, $0<$ $\nu_{A}<1$.

$p_{A}^{(i)}$ Alice's belief on the likelihood of E's occurring in round $i$ after she observes Bob's most recent report and before she takes any actions in that round, taking into account actions in future rounds.

$p_{B}^{(i)} B$ ob's belief on the likelihood of $E$ 's occurring in round $i$ after he observes Alice's most recent report and before he takes any actions in that round, taking into account actions in future rounds.

$\rho_{e}^{(i)}(\cdot)$ function of payoff scores of efforts devoted in round $i$.

$\rho_{s}^{(i)}(\cdot)$ function of payoff scores earned from moving the probability in prediction market in round $i$ if $E$ occurs (succeeds) after $T$ rounds.

$\rho_{f}^{(i)}(\cdot)$ function of payoff scores earned from moving the probability in prediction market in round $i$ if $E$ does not occur (fails) after $T$ rounds.

$\delta$ discounting factor on the future profits, $0<\delta<1$. 
$\pi_{A}^{(i)}$ payoff scores earned by $A$ lice from round $i$ to round $T$, including scores earned in this round and discounting future scores.

$\pi_{B}^{(i)}$ payoff scores earned by $B$ ob from round $i$ to round $T$, including scores earned in this round and discounting future scores.

$I_{A}^{(i)}$ the system state that Alice has in round $i$, after she observes Bob's most recent report in the prediction market, and before she takes any actions in that round.

$\mathbb{E}\left[\pi_{A}^{(i)}\left(I_{A}^{(i)}, a_{A}^{(i)}\right)\right]$ the expected value of $\pi_{A}^{(i)}$ if the current state is $I_{A}^{(i)}$ and the current round's action set is $a_{A}^{(i)}$.

$\mathbb{E}\left[\pi_{A}^{(i) *}\left(I_{A}^{(i)}, a_{A}^{(i)}\right)\right]$ the optimal expected value of $\pi_{A}^{(i)}$ given the current state $I_{B}^{(i)}$.

$I_{B}^{(i)}$ the system state that $B$ ob has in round $i$, after he observes Alice's most recent report in the prediction market, and before he takes any actions in that round.

$\mathbb{E}\left[\pi_{B}^{(i)}\left(I_{B}^{(i)}, a_{B}^{(i)}\right)\right]$ the expected value of $\pi_{B}^{(i)}$ given if the current state is $I_{B}^{(i)}$ and the current round's action set is $a_{B}^{(i)}$.

$\mathbb{E}\left[\pi_{B}^{(i) *}\left(I_{B}^{(i)}, a_{B}^{(i)}\right)\right]$ the optimal expected value of $\pi_{B}^{(i)}$ given the current state $I_{B}^{(i)}$. 